\title{
On the Existence and Uniqueness of the Eigenvalue Decomposition of a Parahermitian Matrix
}

\author{
Stephan Weiss ${ }^{(1)}$, Senior Member, IEEE, Jennifer Pestana ${ }^{(1)}$, and Ian K. Proudler
}

\begin{abstract}
This paper addresses the extension of the factorization of a Hermitian matrix by an eigenvalue decomposition (EVD) to the case of a parahermitian matrix that is analytic at least on an annulus containing the unit circle. Such parahermitian matrices contain polynomials or rational functions in the complex variable $z$ and arise, e.g., as cross spectral density matrices in broadband array problems. Specifically, conditions for the existence and uniqueness of eigenvalues and eigenvectors of a parahermitian matrix EVD are given, such that these can be represented by a power or Laurent series that is absolutely convergent, at least on the unit circle, permitting a direct realization in the time domain. Based on an analysis of the unit circle, we prove that eigenvalues exist as unique and convergent but likely infinite-length Laurent series. The eigenvectors can have an arbitrary phase response and are shown to exist as convergent Laurent series if eigenvalues are selected as analytic functions on the unit circle, and if the phase response is selected such that the eigenvectors are Hölder continuous with $\alpha>\frac{1}{2}$ on the unit circle. In the case of a discontinuous phase response or if spectral majorisation is enforced for intersecting eigenvalues, an absolutely convergent Laurent series solution for the eigenvectors of a parahermitian EVD does not exist. We provide some examples, comment on the approximation of a parahermitian matrix EVD by Laurent polynomial factors, and compare our findings to the solutions provided by polynomial matrix EVD algorithms.
\end{abstract}

\section{INTRODUCTION}

$\mathbf{F}$ OR a multi-channel signal $\mathbf{x}[n] \in \mathbb{C}^{M}$ the instantaneous covariance matrix is $\mathbf{R}=\mathcal{E}\left\{\mathbf{x}[n] \mathbf{x}^{\mathrm{H}}[n]\right\}$, where $\mathcal{E}\{\cdot\}$ denotes the expectation operator and $\mathrm{x}^{\mathrm{H}}$ represents the Hermitian transpose of $\mathbf{x}$. It captures the correlation and phase information on which rests the optimal solution of many narrowband array processing problems. For broadband signals, explicit delays must be considered instead of phase shifts, and capturing

Manuscript received August 31, 2017; revised December 31, 2017 and February 16, 2018; accepted February 20, 2018. Date of publication March 6, 2018; date of current version April 17, 2018. The associate Editor coordinating the review of this manuscript and approving it for publication was Dr. Xavier Mestre. This work was supported in part by the Engineering and Physical Sciences Research Council, under Grant EP/K014307/1 and the MOD University Defence Research Collaboration in Signal Processing. (Corresponding author: Stephan Weiss.)

S. Weiss is with the Centre for Signal and Image Processing, Department of Electronic and Electrical Engineering, University of Strathclyde, Glasgow G1 1XW, U.K. (e-mail: stephan.weiss@strath.ac.uk).

J. Pestana is with the Department of Mathematics and Statistics, University of Strathclyde, Glasgow G1 1XW, U.K. (e-mail: jennifer.pestana@ strath.ac.uk).

I. K. Proudler is with the Centre for Signal and Image Processing, Department of Electronic and Electrical Engineering, University of Strathclyde, Glasgow G1 1XW, U.K., and also with the School for Mechanical, Electrical and Manufacturing Engineering, Loughborough University, Leicestershire LE11 3TU, U.K. (e-mail: ian.proudler@strath.ac.uk).

Color versions of one or more of the figures in this paper are available online at http://ieeexplore.ieee.org.

Digital Object Identifier 10.1109/TSP.2018.2812747 the second order statistics can be accomplished via the spacetime covariance matrix $\mathbf{R}[\tau]=\mathcal{E}\left\{\mathbf{x}[n] \mathbf{x}^{\mathrm{H}}[n-\tau]\right\}$ with a discrete lag parameter $\tau$. Since $\mathbf{R}[\tau]$ contains auto- and crosscorrelation terms of $\mathbf{x}[n]$ it follows that $\mathbf{R}[\tau]=\mathbf{R}^{\mathrm{H}}[-\tau]$. Taking the $z$-transform leads to the cross spectral density (CSD) matrix ${ }^{1} \boldsymbol{R}(z)=\sum_{\tau} \mathbf{R}[\tau] z^{-\tau}$, which is a function of the complex variable $z$. The CSD matrix satisfies the parahermitian property $\boldsymbol{R}(z)=\boldsymbol{R}^{\mathrm{P}}(z)$, where the parahermitian operation $\boldsymbol{R}^{\mathrm{P}}(z)=\boldsymbol{R}^{\mathrm{H}}\left(1 / z^{*}\right)$ involves Hermitian transposition and time reversal [1]. We call any $\mathbf{R}(z)$ satisfying the parahermitian property a parahermitian matrix.

In the narrowband case, many optimal and robust solutions to signal processing problems rely on matrix decompositions [2], [3], particularly on the eigenvalue decomposition (EVD) of $\mathbf{R}$. To extend the utility of the EVD to the broadband case requires an equivalent factorisation of the parahermitian matrix $\boldsymbol{R}(z)$. Under the restriction of $\boldsymbol{R}(z)$ having Laurent polynomial entries, a number of algorithms have been reported in the literature over the past decade [4]-[12] that calculate an approximate polynomial EVD $\boldsymbol{R}(z) \approx \hat{\boldsymbol{U}}(z) \hat{\boldsymbol{\Gamma}}(z) \hat{\boldsymbol{U}}^{\mathrm{P}}(z)$ consisting of Laurent polynomial factors, where $\hat{\boldsymbol{U}}(z)$ is a paraunitary matrix, that is, $\hat{\boldsymbol{U}}(z)$ satisfies $\hat{\boldsymbol{U}}^{-1}(z)=\hat{\boldsymbol{U}}^{\mathrm{P}}(z)[1]$ and $\hat{\boldsymbol{\Gamma}}(z)$ is a diagonal polynomial matrix containing power spectral density (PSD) terms.

The above polynomial matrix EVD algorithms have proved useful in a number of applications, for example in denoisingtype [13] or decorrelating array preprocessors [14], transmit and receive beamforming across broadband MIMO channels [15] [17], broadband angle of arrival estimation [18], [19], optimum subband partitioning of beamformers [20], filter bank-based channel coding [21], fixed [22] and adaptive (i.e. minimum variance distortionless response) broadband beamforming [23], and blind source separation [24]. The polynomial approach can enable solutions that otherwise have been unobtainable: e.g. the design of optimal compaction filter banks beyond the two channel case [8], the coherent estimation of broadband sources [19] without side-information, or the decoupling of dimensions and hence reduction of computational complexities of the quiescent beamformer, the blocking matrix and the adaptive noise canceller in a polynomial generalised sidelobe canceller [23].

\footnotetext{
${ }^{1}$ In our notation, boldface upper and lower case font refers to matrices and vectors, respectively. A bold A generally refers to a time domain quantity, while $\boldsymbol{A}(z)$ is a transform domain quantity, with $\boldsymbol{A}\left(\mathrm{e}^{\mathrm{j} \Omega}\right)$ typically its evaluation on the unit circle, $z=\mathrm{e}^{\mathrm{j} \Omega}$
} 
Despite the numerous algorithms ${ }^{2}$ and a number of successful applications, the theoretical foundations of the existence and uniqueness of a polynomial EVD have received little attention. The book by Gohberg et al. [25] considers the related factorisation of a self-adjoint matrix $\boldsymbol{A}(x)=\boldsymbol{A}^{\mathrm{H}}(x), x \in \mathbb{R}$, which can be applied to a parahermitian matrix $\boldsymbol{R}(z)$ on the unit circle via the reparameterisation $z=\mathrm{e}^{\mathrm{j} \Omega}$. Decompositions such as the Smith and Smith-MacMillan forms for matrices of polynomials and rational functions, respectively, are proven to exist [1], [25], but do not involve paraunitary and parahermitian factors as required for the polynomial EVD in [4], [6]. Significantly, Icart and Comon [26] prove, based on known decompositions and the Stone-Weierstrass theorem, that the decomposition factors of a positive semi-definite parahermitian matrix can be approximated by Laurent polynomials. For the polynomial EVD in [9] and the related problem of a Laurent-polynomial QR decomposition [27], [28], the authors show that the factor matrices generally cannot exist as Laurent polynomials because the required solutions involve divisions and square root operations of polynomials.

This paper aims to extend the work in [26] and to clarify the existence and uniqueness of factorising a parahermitian $\boldsymbol{R}(z)$ into paraunitary and diagonal parahermitian matrices. We generalise $\boldsymbol{R}(z)$ to include not just polynomials but rational functions in $z \in \mathbb{C}$. Since the EVD even for a polynomial $\boldsymbol{R}(z)$ is not guaranteed to exist with Laurent polynomial factors [26], we refer to the decomposition $\boldsymbol{R}(z)=\boldsymbol{U}(z) \boldsymbol{\Gamma}(z) \boldsymbol{U}^{\mathrm{P}}(z)$, with absolutely convergent Laurent series ${ }^{3} \boldsymbol{U}(z)$ and $\boldsymbol{\Gamma}(z)$ as a parahermitian matrix EVD (PhEVD). If it exists, the matrix of eigenvalues, $\boldsymbol{\Gamma}(z)$, is parahermitian and a Laurent series; the eigenvectors in $\boldsymbol{U}(z)$ may exist as Laurent series, and, if causal, may even be power series. Absolute convergence-in some cases analyticity - of these factors will ensure that these Laurent or power series permit a direct time domain realisation. Our proof of existence and uniqueness proceeds in two stages. First, we characterize the PhEVD of $\boldsymbol{R}(z)$ on the unit circle, i.e. in terms of the normalised angular frequency $\Omega \in \mathbb{R}$. We next state the conditions that must be satisfied for the PhEVD factors to be representable as Laurent or power series. The main thrust of our analysis rests (i) on the analyticity of $\boldsymbol{R}(z)$ [29] to guarantee that the CSD matrix is entirely characterised by its evaluation on the unit circle, (ii) on matrix perturbation theory [30], [31] to demonstrate the smooth evolution of EVD factors as functions of $\Omega$, and (iii) on complex function analysis [32] to extract convergent Laurent or power series. Throughout we assume that any eigenvalues of $\boldsymbol{R}(z)$ are non-negative for all $|z|=1$ and that $\boldsymbol{R}(z)$ is analytic at least on an annulus containing the unit circle.

The paper is organised as follows. Section II provides background on the existence and uniqueness of the EVD of a

\footnotetext{
${ }^{2}$ Many of these algorithms have convergence proofs, even though it is not clear to which matrices they converge.

${ }^{3}$ The infinite sum $\sum_{n} c_{n} z^{-n}$ is a power series for $n \in \mathbb{N}$, while for a Laurent series $n \in \mathbb{Z}$. It does not need to converge to be called a series, but convergence criteria will be discussed later. Polynomials and Laurent polynomials are power and Laurent series, respectively, with a finite number of non-zero coefficients $c_{n}$.
}

Hermitian matrix, and properties of and decomposition algorithms for parahermitian matrices. The parahermitian EVD problem is then mapped to the unit circle, i.e. $|z|=1$, in Section III where we look at the conditions under which a function of frequency admits a time series representation. We then apply these results to the frequency domain EVD. This is first addressed in Section IV for the easier case that $\boldsymbol{R}(z)$ has eigenvalues of algebraic multiplicity one for all $|z|=1$, i.e. when the eigenvalues are viewed in the Fourier domain as PSDs that do not overlap. Section V considers the case that $\boldsymbol{R}(z)$ has, at least for some $z$ on the unit circle, eigenvalues of algebraic multiplicity greater than one. It generalises the findings of Section IV and contains the main results of this paper. A numerical example and a comparison with results obtained by iterative polynomial EVD algorithms are provided in Section VI, with concluding remarks in Section VII.

\section{BACKGROUND}

\section{A. Eigenvalue Decomposition}

We restrict $\mathbf{R} \in \mathbb{C}^{M \times M}$ to be positive semi-definite-a property guaranteed if $\mathbf{R}$ is e.g. a covariance matrix or emerges from a product $\mathbf{R}=\mathbf{A} \mathbf{A}^{\mathrm{H}}$, with an arbitrary $\mathbf{A} \in \mathbb{C}^{M \times L}$. For any Hermitian matrix $\mathbf{R}=\mathbf{R}^{\mathrm{H}}$, its eigenvalue decomposition

$$
\mathbf{R}=\mathbf{Q} \Lambda \mathbf{Q}^{\mathrm{H}}
$$

exists, with the diagonal matrix $\boldsymbol{\Lambda}$ containing the real-valued, non-negative eigenvalues $\lambda_{m} \in \mathbb{R}, m=1 \ldots M$, and the eigenvectors $\mathbf{q}_{m} \in \mathbb{C}^{M}$, which we constrain to be orthonormal so that they form the columns of a unitary matrix $\mathbf{Q}$.

While the EVD in (1) has unique eigenvalues, their sequence along the diagonal of $\boldsymbol{\Lambda}$ can be arbitrary. This ambiguity w.r.t. a permutation can be removed by ordering eigenvalues in $\Lambda$ in descending sequence,

$$
\lambda_{1} \geq \lambda_{2} \geq \cdots \geq \lambda_{M} \geq 0 .
$$

In the case of $M$ distinct eigenvalues, the eigenvectors of $\mathbf{R}$ are unique except for a phase rotation. If $\mathbf{q}_{m}$ is the eigenvector that corresponds to the $m$ th eigenvalue $\lambda_{m}$ of $\mathbf{R}$, then

$$
\mathbf{R} \mathbf{q}_{m} \mathrm{e}^{\mathrm{j} \varphi}=\lambda_{m} \mathbf{q}_{m} \mathrm{e}^{\mathrm{j} \varphi}
$$

holds for an arbitrary phase shift $\varphi$. Therefore, if $\mathbf{q}_{m}$ is an $m$ th eigenvector, then so is $\mathbf{q}_{m}^{\prime}=\mathbf{q}_{m} \mathrm{e}^{\mathrm{j} \varphi}$.

Ambiguity w.r.t. the eigenvectors also arises if eigenvalues have an algebraic multiplicity greater than one, i.e. when the eigenvalues are no longer distinct. If $\lambda_{m}=\lambda_{m+1}=$ $\ldots \lambda_{m+C-1}$, these eigenvalues possess an algebraic multiplicity of $C$ and only the $C$-dimensional subspace containing the eigenvectors corresponding to this eigenvalue is uniquely defined, within which the eigenvectors $\mathbf{q}_{m}, \mathbf{q}_{m+1}, \ldots \mathbf{q}_{m+C-1}$ can form an arbitrary orthonormal basis: if $\mathbf{q}_{m}, \ldots \mathbf{q}_{m+C-1}$ are eigenvectors of $\mathbf{R}$, then so are $\mathbf{q}_{m}^{\prime}, \ldots \mathbf{q}_{m+C-1}^{\prime}$,

$$
\left[\mathbf{q}_{m}^{\prime}, \ldots \mathbf{q}_{m+C-1}^{\prime}\right]=\left[\mathbf{q}_{m}, \ldots \mathbf{q}_{m+C-1}\right] \mathbf{V}
$$

for any arbitrary unitary matrix $\mathbf{V} \in \mathbb{C}^{C \times C}$. Note that in the case of distinct eigenvalues with $C=1$, (4) incorporates the phase ambiguity of (3) since $\mathbf{V}$ will be the scalar $\mathrm{e}^{\mathrm{j} \varphi}$. 


\section{B. Parahermitian Space-Time Covariance Matrix}

To understand how a parahermitian matrix may be obtained, we consider a scenario where $L$ independent sources with nonnegative, real power spectral densities (PSD) $S_{\ell}(z), \ell=1 \ldots L$, contribute to $M$ sensor measurements $x_{m}[n], m=1 \ldots M$. If these are organised in a vector $\mathbf{x}[n]=\left[x_{1}[n] \ldots x_{M}[n]\right]^{\mathrm{T}}$, then the space-time covariance matrix is

$$
\mathbf{R}[\tau]=\mathcal{E}\left\{\mathbf{x}[n] \mathbf{x}^{\mathrm{H}}[n-\tau]\right\} .
$$

If the PSD of the $\ell$ th source is generated by a stable and causal innovation filter $F_{\ell}(z)$ [33], and $H_{m \ell}(z)$ describes the transfer function of the causal and stable system between the $\ell$ th source and the $m$ th sensor, then

$$
\boldsymbol{R}(z)=\boldsymbol{H}(z)\left[\begin{array}{lll}
S_{1}(z) & & \\
& \ddots & \\
& & S_{L}(z)
\end{array}\right] \boldsymbol{H}^{\mathrm{P}}(z)
$$

with the element in the $m$ th row and $\ell$ th column of $\boldsymbol{H}(z)$ : $\mathbb{C} \rightarrow \mathbb{C}^{M \times L}$ given by $H_{m \ell}(z)$, and $S_{\ell}(z)=F_{\ell}(z) F^{\mathrm{P}}(z)$ the $\ell$ th element of the diagonal matrix of source PSDs.

The factorisation (6) can include the source model matrix $\boldsymbol{F}(z)=\operatorname{diag}\left\{F_{1}(z), \ldots, F_{L}(z)\right\}: \mathbb{C} \rightarrow \mathbb{C}^{L \times L}$, such that

$$
\boldsymbol{R}(z)=\boldsymbol{H}(z) \boldsymbol{F}(z) \boldsymbol{F}^{\mathrm{P}}(z) \boldsymbol{H}^{\mathrm{P}}(z) .
$$

The components of $\boldsymbol{H}(z)$ and the source model $\boldsymbol{F}(z)$ are assumed to be causal and stable, and their entries can be either polynomials or rational functions in $z$. For the more general latter case, let the maximum modulus of a pole of any component of either $\boldsymbol{H}(z)$ or $\boldsymbol{F}(z)$ be $\rho$, where $0<\rho<1$. Thus the region of convergence for $\boldsymbol{H}(z) \boldsymbol{F}(z)$ is $|z|>\rho$, while for the anticausal term $\boldsymbol{F}^{\mathrm{P}}(z) \boldsymbol{H}^{\mathrm{P}}(z)$ it is $|z|<\rho^{-1}$. Overall, therefore, the CSD matrix $\boldsymbol{R}(z)$ in (7) can be represented as a Laurent series whose convergence region $\mathcal{D}$ is the annulus $\rho<|z|<\rho^{-1}$ [29], [34]. Hence, within this region, all entries of $\boldsymbol{R}(z)$ are analytic and are therefore continuous and infinitely differentiable [29].

Since the PSDs satisfy $S_{\ell}(z)=S_{\ell}^{\mathrm{P}}(z)$, it is evident from both (6) and (7) that $\boldsymbol{R}(z)=\boldsymbol{R}^{\mathrm{P}}(z)$ and so is parahermitian. The EVD of Section II-A can only diagonalise $\mathbf{R}[\tau]$ for one particular lag value $\tau$, typically the Hermitian (narrowband) covariance matrix $\mathbf{R}[0]$. The next section reviews efforts to diagonalise $\boldsymbol{R}(z)$ or, equivalently, diagonalise $\mathbf{R}[\tau]$ for all lags $\tau$.

\section{Polynomial EVD}

A self-adjoint matrix $\boldsymbol{A}(x)$, with $x \in \mathbb{R}$, which satisfies $\boldsymbol{A}(x)=\boldsymbol{A}^{\mathrm{H}}(x)$, has an EVD [30], [35] or spectral factorisation [25], which can therefore describe the EVD of $\boldsymbol{R}(z)$ evaluated on the unit circle, but not an EVD of $\boldsymbol{R}(z)$ itself. The first mention of a polynomial EVD is in [4], which also proposed the second order sequential best rotation (SBR2) algorithm for its iterative approximation using Laurent polynomials. Over the past decade a number of algorithms have emerged [4], [6]-[12], [36], which share the restriction of considering the EVD of a parahermitian matrix $\boldsymbol{R}(z)$ whose elements are Laurent polynomials. In cases where the support is unknown or the source model in (7) contains rational functions, the auto- and cross- correlation sequences in (5) may be estimated or approximated over a finite window of lags [8].

The polynomial EVD or McWhirter decomposition in [6] is stated as ${ }^{4}$

$$
\boldsymbol{R}(z) \approx \hat{\boldsymbol{U}}(z) \hat{\boldsymbol{\Gamma}}(z) \hat{\boldsymbol{U}}^{\mathrm{P}}(z)
$$

where the elements of the matrices on the r.h.s. are Laurent polynomials, $\hat{\boldsymbol{U}}(z)$ is paraunitary and $\hat{\boldsymbol{\Gamma}}(z)$ is diagonal and spectrally majorised, such that for the PSDs along the main diagonal,

$$
\hat{\gamma}_{m}\left(\mathrm{e}^{\mathrm{j} \Omega}\right) \geq \hat{\gamma}_{m+1}\left(\mathrm{e}^{\mathrm{j} \Omega}\right) \forall \Omega,
$$

for $m=1 \ldots(M-1)$. Even though the term 'polynomial EVD' is not mentioned in [37], diagonalisation and spectral majorisation were introduced there in the context of optimising filter banks w.r.t. subband coding gain.

The approximation sign in the McWhirter decomposition (8) has been included in all subsequent algorithm designs over the past decade. Even though many algorithms can be proven to converge, in the sense that off-diagonal energy of $\Gamma(z)$ is reduced at each iteration, see e.g. [6], [8], [10]-[12], and there is no practical experience yet where algorithms could not find a practicable factorisation, the only work towards the existence of the polynomial EVD has been reported in [26]. However, this provides limited understanding under which circumstances existence is guaranteed and does not address the uniqueness or ambiguity of eigenvalues and eigenvectors.

\section{PARAhERmitian Matrix EVD}

We first focus on the task of identifying the eigenvalues of a parahermitian matrix in the Fourier domain, and are particularly interested in determining how smoothly these vary, before investigating the corresponding eigenvectors. Our approach rests on the conditions under which a function on the unit circle (i.e. a function of frequency) admits an absolutely convergent power or Laurent series, or even permits an analytic continuation to $z \in \mathbb{C}$, the ultimate aim being to find a suitable representation in the time domain.

\section{A. EVD on the Unit Circle}

We assume that the parahermitian matrix $\boldsymbol{R}(z): \mathbb{C} \rightarrow$ $\mathbb{C}^{M \times M}$ contains Laurent polynomials or rational functions in $z$, and is analytic in the annulus $\mathcal{D}=\left\{z: z \in \mathbb{C}, \rho<|z|<\rho^{-1}\right\}$ with $0<\rho<1$, as motivated in Section II-B. Since the unit circle is included in $\mathcal{D}$, it follows from Cauchy's integral formula that every value of $\boldsymbol{R}(z)$ for $\rho<|z|<1$ is specified by its values for $|z|=1$ [34]. Because of the parahermitian property $\boldsymbol{R}(z)=\boldsymbol{R}^{\mathrm{H}}\left(1 / z^{*}\right)$, every value of $\boldsymbol{R}(z)$ for $1>|z|>\rho^{-1}$ is also specified by the values of $\boldsymbol{R}(z)$ for $|z|=1$. From a practical aspect, the inverse $z$-transform requires evaluation on a closed path in $\mathcal{D}$, which here can be the unit circle. This inverse transform leads back to the time domain, which then implies the

\footnotetext{
${ }^{4}$ The McWhirter decomposition in [6] is defined with the parahermitian $\hat{\boldsymbol{U}}^{\mathrm{P}}(z)$ instead of $\hat{\boldsymbol{U}}(z)$. W.l.g. and for consistency with (1), we use the notation in (8).
} 
existence of a Laurent series $\boldsymbol{R}(z)=\sum_{\tau} \mathbf{R}[\tau] z^{-\tau}$ : thus $\boldsymbol{R}(z)$ is recovered from $\boldsymbol{R}\left(\mathrm{e}^{\mathrm{j} \Omega}\right)$. Therefore, in $\mathcal{D}, \boldsymbol{R}(z)$ is uniquely characterised by $\boldsymbol{R}\left(\mathrm{e}^{\mathrm{j} \Omega}\right)=\boldsymbol{R}(z)_{z=\mathrm{e}^{\mathrm{j} \Omega}}$ and vice versa, where $\boldsymbol{R}\left(\mathrm{e}^{\mathrm{j} \Omega}\right)$ is Hermitian, $\boldsymbol{R}\left(\mathrm{e}^{\mathrm{j} \Omega}\right)=\boldsymbol{R}^{\mathrm{H}}\left(\mathrm{e}^{\mathrm{j} \Omega}\right) \forall \Omega$.

An EVD of $\boldsymbol{R}\left(\mathrm{e}^{\mathrm{j} \Omega}\right)$ can be evaluated at every point along the continuous normalised angular frequency variable $\Omega$, such that

$$
\boldsymbol{R}\left(\mathrm{e}^{\mathrm{j} \Omega}\right)=\boldsymbol{Q}\left(\mathrm{e}^{\mathrm{j} \Omega}\right) \boldsymbol{\Lambda}\left(\mathrm{e}^{\mathrm{j} \Omega}\right) \boldsymbol{Q}^{\mathrm{H}}\left(\mathrm{e}^{\mathrm{j} \Omega}\right) .
$$

At any arbitrary frequency $\Omega$, the properties of the EVD in (1) apply equally to (9), with the existence and uniqueness of its eigenvalues and -vectors as discussed in Section II-A. Since a parahermitian matrix is Hermitian on the unit circle it has real eigenvalues there.

\section{B. Time-Domain Realisation}

We want to form matrix functions $\boldsymbol{U}(z)$ and $\boldsymbol{\Gamma}(z)$ as this will lead to a time domain representation and hence allow them to be implemented. The matrix functions $\boldsymbol{U}(z)$ and $\boldsymbol{\Gamma}(z)$ need to match $\boldsymbol{Q}\left(\mathrm{e}^{\mathrm{j} \Omega}\right)$ and $\boldsymbol{\Lambda}\left(\mathrm{e}^{\mathrm{j} \Omega}\right)$ in (9) at every frequency. There are infinitely many ways to do this. However we require a mechanism that allows us to extract a direct realisation in the time domain of these functions on the unit circle i.e. $\Lambda\left(\mathrm{e}^{\mathrm{j} \Omega}\right) \rightarrow \boldsymbol{\Gamma}[\tau]$ and $\boldsymbol{Q}\left(\mathrm{e}^{\mathrm{j} \Omega}\right) \rightarrow \mathbf{U}[n]$, and this restricts the acceptable choices of $\boldsymbol{U}(z)$ and $\boldsymbol{\Gamma}(z)$. Ideally, we would like to extract analytic functions $\boldsymbol{\Gamma}(z)$ and $\boldsymbol{U}(z)$, but we will be content if they can be represented by absolutely convergent power or Laurent series at least on the unit circle. Within their region of convergence, these functions $\boldsymbol{\Gamma}(z)$ and $\boldsymbol{U}(z)$ are guaranteed to be unique [38], [39].

If an arbitrary $2 \pi$-periodic function $X\left(\mathrm{e}^{\mathrm{j} \Omega}\right): \mathbb{R} \rightarrow \mathbb{C}$ has only a finite number of discontinuities, we can write $X\left(\mathrm{e}^{\mathrm{j} \Omega}\right)=$ $\sum_{n} x[n] \mathrm{e}^{\mathrm{j} \Omega n}$. For the Fourier coefficients $x[n]$ to represent an absolutely convergent Laurent or power series, we require absolute summability, i.e. $\sum_{n}|x[n]|<\infty$. A sufficient condition for this is to restrict $X\left(\mathrm{e}^{\mathrm{j} \Omega}\right)$ to be Hölder continuous with $\alpha>\frac{1}{2}[40]$, such that

$$
\sup _{\Omega_{1}, \Omega_{2} \in \mathbb{R}}\left|X\left(\mathrm{e}^{\mathrm{j} \Omega_{1}}\right)-X\left(\mathrm{e}^{\mathrm{j} \Omega_{2}}\right)\right| \leq C\left|\mathrm{e}^{\mathrm{j} \Omega_{1}}-\mathrm{e}^{\mathrm{j} \Omega_{2}}\right|^{\alpha}
$$

with some $C \in \mathbb{R}$. A continuous function $X\left(\mathrm{e}^{\mathrm{j} \Omega}\right)$ is Hölder continuous if it does not behave too 'wildly'. For the remainder of the paper, Hölder continuity always implies the condition $\alpha>\frac{1}{2}$. In this case, the time domain realisation can be obtained by the inverse Fourier transform

$$
x[n]=\frac{1}{2 \pi} \int_{-\pi}^{\pi} X\left(\mathrm{e}^{\mathrm{j} \Omega}\right) \mathrm{e}^{\mathrm{j} \Omega n} \mathrm{~d} \Omega .
$$

If, moreover, $X\left(\mathrm{e}^{\mathrm{j} \Omega}\right)$ is analytic then we know that we can apply the inverse $z$-transform

$$
x[n]=\frac{1}{2 \pi \mathrm{j}} \oint_{\mathcal{C}} X(z) z^{n} \frac{\mathrm{d} z}{z}
$$

for $\mathcal{C}$ a closed counter-clockwise curve in the region of convergence of $X(z)$. Choosing $\mathcal{C}$ to be the unit circle the inverse $z$-transform becomes the inverse Fourier transform (11). Hence in this case the inverse Fourier transform can lead to a Laurent series $X(z)=\sum_{n} x[n] z^{-n}$ that is valid in an annulus with nonempty interior containing the unit circle. More generally, we can define $X(z)=\sum_{n} x[n] z^{n},|z|=1$ from the Fourier series and attempt to analytically continue this representation. However, the region of convergence of the resulting series is difficult to determine.

Throughout, we use the terms "absolutely convergent power series" and "absolutely convergent Laurent series" to represent a power (Laurent) series that is absolutely convergent, at least on the unit circle. While there appears to be no simple necessary condition for $X\left(\mathrm{e}^{\mathrm{j} \Omega}\right)$ to yield an absolutely convergent power or Laurent series $x[n]$, a discontinuous $X\left(\mathrm{e}^{\mathrm{j} \Omega}\right)$ is sufficient to exclude the existence of an absolutely convergent Fourier series and hence of an absolutely convergent power or Laurent series $x[n]$ [40]. In general for the case of continuity, Weierstrass [41], [42] guarantees uniform convergence of a series of functions. These functions may change with the approximation order, hence it is neither possible to state a limit for infinite order using a power series, nor to obtain an approximation by truncation of that power series. Its use in this context is therefore limited. Therefore, the arguments in the remainder of this paper will focus on the Hölder continuity and potential smoothness of the factors $\boldsymbol{Q}\left(\mathrm{e}^{\mathrm{j} \Omega}\right)$ and $\boldsymbol{\Lambda}\left(\mathrm{e}^{\mathrm{j} \Omega}\right)$ in (9).

\section{Continuity of Eigenvalues}

We now inspect how smoothly eigenvalues $\lambda_{m}\left(\mathrm{e}^{\mathrm{j} \Omega}\right), m=$ $1 \ldots M$, of $\boldsymbol{R}\left(\mathrm{e}^{\mathrm{j} \Omega}\right)$ in (9) evolve with the frequency $\Omega$. To quantify the change that is induced in the eigenvalues of $\boldsymbol{R}\left(\mathrm{e}^{\mathrm{j}(\Omega+\Delta \Omega)}\right)$, with $\Delta \Omega$ a small change in frequency, perturbation theory for matrices [30], [31] provides some useful results. The Hoffman-Wielandt theorem [43] shows that ${ }^{5}$

$$
\sum_{i}\left|\lambda_{i}\left(\mathrm{e}^{\mathrm{j} \Omega}\right)-\lambda_{i}\left(\mathrm{e}^{\mathrm{j}(\Omega+\Delta \Omega)}\right)\right| \leq\left\|\boldsymbol{R}\left(\mathrm{e}^{\mathrm{j} \Omega}\right)-\boldsymbol{R}\left(\mathrm{e}^{\mathrm{j}(\Omega+\Delta \Omega)}\right)\right\|_{\mathrm{F}},
$$

assuming that the eigenvalues are ordered, with $\|\cdot\|_{\mathrm{F}}$ the Frobenius norm. Since based on the source model in Section II-B, $\boldsymbol{R}(z)$ is analytic and hence continuous,

$$
\lim _{\Delta \Omega \rightarrow 0}\left\|\boldsymbol{R}\left(\mathrm{e}^{\mathrm{j} \Omega}\right)-\boldsymbol{R}\left(\mathrm{e}^{\mathrm{j}(\Omega+\Delta \Omega)}\right)\right\|_{\mathrm{F}}=0,
$$

which also implies continuity of $\lambda_{m}\left(\mathrm{e}^{\mathrm{j} \Omega}\right), m=1 \ldots M$, because

$$
\lim _{\Delta \Omega \rightarrow 0} \sum_{i}\left|\lambda_{i}\left(\mathrm{e}^{\mathrm{j} \Omega}\right)-\lambda_{i}\left(\mathrm{e}^{\mathrm{j}(\Omega+\Delta \Omega)}\right)\right|=0
$$

must also hold on the 1.h.s. of (12). Beyond continuity, analyticity of $\mathbf{R}(z)$ on an annulus containing the unit circle ensures that the eigenvalues $\lambda_{1}\left(\mathrm{e}^{\mathrm{j} \Omega}\right), \ldots, \lambda_{M}\left(\mathrm{e}^{\mathrm{j} \Omega}\right)$ can be chosen to be analytic for $\Omega \in \mathbb{R}$ [30], [35], and therefore can also be infinitely differentiable. ${ }^{6}$

\footnotetext{
${ }^{5}$ A regular perturbation of $\boldsymbol{R}(z)$ can lead to either a regular or singular perturbation of the eigenvalues, but we are here only interested in the continuity of the latter.

${ }^{6}$ A similar frequency-domain approach for arbitrary matrices exists with the analytic singular value decomposition [44], [45].
} 


\section{Invariant Subspaces and Subspace Distance}

Having characterized the eigenvalues of $\boldsymbol{R}(z)$, we now turn to their corresponding eigenvectors, reviewing the effect of matrix perturbations on eigenvector subspaces. As highlighted in Section II-A, eigenvectors corresponding to multiple eigenvalues are not unique, and even though eigenvectors corresponding to tightly clustered eigenvalues are very likely ill-conditioned, the subspace spanned by these eigenvectors is usually insensitive to perturbations [31], [46].

Assume a cluster of $C$ adjacent (potentially multiple) eigenvalues organised in the diagonal matrix $\boldsymbol{\Lambda}_{1}\left(\mathrm{e}^{\mathrm{j} \Omega}\right): \mathbb{R} \rightarrow \mathbb{R}^{C \times C}$, with $\boldsymbol{\Lambda}_{2}\left(\mathrm{e}^{\mathrm{j} \Omega}\right): \mathbb{R} \rightarrow \mathbb{R}^{(M-C) \times(M-C)}$ containing the remaining $M-C$ eigenvalues. The spread of the cluster is assumed to be small compared to the distance $\delta$ to the next-nearest eigenvalue outside this cluster [3], i.e.

$$
\begin{gathered}
\max _{\lambda_{i}, \lambda_{j} \in \boldsymbol{\Lambda}_{1}\left(\mathrm{e}^{\mathrm{j} \Omega}\right)}\left|\lambda_{i}-\lambda_{j}\right| \ll \min _{\lambda_{1} \in \boldsymbol{\Lambda}_{1}\left(\mathrm{e}^{\mathrm{j} \Omega}\right)}\left|\lambda_{1}-\lambda_{2}\right| \equiv \delta>0 . \\
\lambda_{2} \in \boldsymbol{\Lambda}_{2}\left(\mathrm{e}^{\mathrm{j} \Omega}\right)
\end{gathered}
$$

The parameter $\delta$ in (14) defines the spectral distance between the eigenvalues in $\boldsymbol{\Lambda}_{1}\left(\mathrm{e}^{\mathrm{j} \Omega}\right)$ and in $\boldsymbol{\Lambda}_{2}\left(\mathrm{e}^{\mathrm{j} \Omega}\right)$. If $\boldsymbol{Q}_{1}\left(\mathrm{e}^{\mathrm{j} \Omega}\right): \mathbb{R} \rightarrow$ $\mathbb{C}^{M \times C}$ is a matrix whose columns are formed by the $C$ eigenvectors of $\boldsymbol{R}\left(\mathrm{e}^{\mathrm{j} \Omega}\right)$ corresponding to $\boldsymbol{\Lambda}_{1}\left(\mathrm{e}^{\mathrm{j} \Omega}\right)$, and $\boldsymbol{Q}_{2}\left(\mathrm{e}^{\mathrm{j} \Omega}\right)$ holds the remaining $M-C$ eigenvectors, we re-organise the EVD as

$$
\boldsymbol{Q}^{\mathrm{H}}\left(\mathrm{e}^{\mathrm{j} \Omega}\right) \boldsymbol{R}\left(\mathrm{e}^{\mathrm{j} \Omega}\right) \boldsymbol{Q}\left(\mathrm{e}^{\mathrm{j} \Omega}\right)=\operatorname{diag}\left\{\boldsymbol{\Lambda}_{1}\left(\mathrm{e}^{\mathrm{j} \Omega}\right), \boldsymbol{\Lambda}_{2}\left(\mathrm{e}^{\mathrm{j} \Omega}\right)\right\},
$$

with $\boldsymbol{Q}\left(\mathrm{e}^{\mathrm{j} \Omega}\right)=\left[\boldsymbol{Q}_{1}\left(\mathrm{e}^{\mathrm{j} \Omega}\right), \boldsymbol{Q}_{2}\left(\mathrm{e}^{\mathrm{j} \Omega}\right)\right]$. Note that in accordance with (4), eigenvectors can have arbitrary phase shifts, which however does not affect the subspace analysis below.

If $\boldsymbol{R}\left(\mathrm{e}^{\mathrm{j} \Omega}\right)$ is perturbed by an increment in frequency, $\Delta \Omega$, then [3], [31]

$$
\begin{aligned}
& \boldsymbol{Q}^{\mathrm{H}}\left(\mathrm{e}^{\mathrm{j} \Omega}\right)\left(\boldsymbol{R}\left(\mathrm{e}^{\mathrm{j}(\Omega+\Delta \Omega)}\right)-\boldsymbol{R}\left(\mathrm{e}^{\mathrm{j} \Omega}\right)\right) \boldsymbol{Q}\left(\mathrm{e}^{\mathrm{j} \Omega}\right)= \\
& {\left[\begin{array}{ll}
\underbrace{\mathbf{E}_{11}\left(\mathrm{e}^{\mathrm{j} \Omega}, \Delta \Omega\right)}_{C} & \begin{array}{l}
\mathbf{E}_{21}^{\mathrm{H}}\left(\mathrm{e}^{\mathrm{j} \Omega}, \Delta \Omega\right) \\
\mathbf{E}_{21}\left(\mathrm{e}^{\mathrm{j} \Omega}, \Delta \Omega\right)
\end{array} \\
\underbrace{\mathbf{E}_{22}\left(\mathrm{e}^{\mathrm{j} \Omega}, \Delta \Omega\right)}_{M-C}
\end{array}\right]}
\end{aligned}
$$

If the increment $\Delta \Omega$ is selected such that [3]

$$
\left\|\boldsymbol{R}\left(\mathrm{e}^{\mathrm{j}(\Omega+\Delta \Omega)}\right)-\boldsymbol{R}\left(\mathrm{e}^{\mathrm{j} \Omega}\right)\right\|_{\mathrm{F}} \leq \frac{\delta}{5},
$$

i.e. such that the perturbation is small compared to the spectral distance $\delta$, then for the two subspaces $\mathcal{Q}_{1}\left(\mathrm{e}^{\mathrm{j} \Omega}\right)=$ $\operatorname{range}\left\{\boldsymbol{Q}_{1}\left(\mathrm{e}^{\mathrm{j} \Omega}\right)\right\}$ and $\mathcal{Q}_{1}\left(\mathrm{e}^{(\mathrm{j} \Omega+\Delta \Omega)}\right)=\operatorname{range}\left\{\boldsymbol{Q}_{1}\left(\mathrm{e}^{\mathrm{j}(\Omega+\Delta \Omega)}\right)\right\}$

$$
\operatorname{dist}\left\{\mathcal{Q}_{1}\left(\mathrm{e}^{\mathrm{j} \Omega}\right), \mathcal{Q}_{1}\left(\mathrm{e}^{\mathrm{j}(\Omega+\Delta \Omega)}\right)\right\} \leq \frac{4}{\delta}\left\|\mathbf{E}_{21}\left(\mathrm{e}^{\mathrm{j} \Omega}, \Delta \Omega\right)\right\|_{\mathrm{F}} .
$$

The distance metric in (18) is defined as

$$
\begin{aligned}
\operatorname{dist}\left\{\mathcal{Q}_{1}\left(\mathrm{e}^{\mathrm{j} \Omega}\right), \mathcal{Q}_{1}\left(\mathrm{e}^{\mathrm{j}(\Omega+\Delta \Omega)}\right)\right\} & =\left\|\boldsymbol{\Pi}_{1}\left(\mathrm{e}^{\mathrm{j} \Omega}\right)-\boldsymbol{\Pi}_{1}\left(\mathrm{e}^{\mathrm{j}(\Omega+\Delta \Omega)}\right)\right\|_{2} \\
& =\sigma_{\max }
\end{aligned}
$$

where $\|\cdot\|_{2}$ is the spectral norm and $\boldsymbol{\Pi}_{1}\left(\mathrm{e}^{\mathrm{j} \Omega}\right)=$ $\boldsymbol{Q}_{1}\left(\mathrm{e}^{\mathrm{j} \Omega}\right) \boldsymbol{Q}_{1}^{\mathrm{H}}\left(\mathrm{e}^{\mathrm{j} \Omega}\right)$ is the projection matrix onto the subspace $\mathcal{Q}_{1}\left(\mathrm{e}^{\mathrm{j} \Omega}\right)$ with $0 \leq \sigma_{\max } \leq 1[3]$.

Because of the continuity of $\boldsymbol{R}\left(\mathrm{e}^{\mathrm{j} \Omega}\right)$ (see (13)) and the unitary invariance of the Frobenius norm, from (16) it follows that $\left\|\mathbf{E}_{21}\left(\mathrm{e}^{\mathrm{j} \Omega}, \Delta \Omega\right)\right\|_{\mathrm{F}} \longrightarrow 0$ as $\Delta \Omega \longrightarrow 0$. Hence the subspace evolves continuously. Interestingly, the distance between the subspaces spanned by $\boldsymbol{Q}_{1}\left(\mathrm{e}^{\mathrm{j} \Omega}\right)$ and $\boldsymbol{Q}_{1}\left(\mathrm{e}^{\mathrm{j}(\Omega+\Delta \Omega)}\right)$ according to (18) is limited by the product of the perturbation-related term $\left\|\mathbf{E}_{21}\left(\mathrm{e}^{\mathrm{j} \Omega}, \Delta \Omega\right)\right\|_{\mathrm{F}}$ and $\delta^{-1}$. Therefore the subspace distance can increase as the distance $\delta$ to the nearest eigenvalue outside the cluster decreases.

\section{E. Eigenvalue Considerations}

The discussion in this section shows that different cases will arise depending on how we choose $\Lambda\left(\mathrm{e}^{\mathrm{j} \Omega}\right)$ and $\boldsymbol{Q}\left(\mathrm{e}^{\mathrm{j} \Omega}\right)$. An arbitrary frequency-dependent and potentially discontinuous permutation $\boldsymbol{P}\left(\mathrm{e}^{\mathrm{j} \Omega}\right)$ can be introduced into (9), such that

$$
\begin{gathered}
\boldsymbol{R}\left(\mathrm{e}^{\mathrm{j} \Omega}\right)=\boldsymbol{Q}\left(\mathrm{e}^{\mathrm{j} \Omega}\right) \boldsymbol{P}^{\mathrm{H}}\left(\mathrm{e}^{\mathrm{j} \Omega}\right) \boldsymbol{P}\left(\mathrm{e}^{\mathrm{j} \Omega}\right) \boldsymbol{\Lambda}\left(\mathrm{e}^{\mathrm{j} \Omega}\right) \boldsymbol{P}^{\mathrm{H}}\left(\mathrm{e}^{\mathrm{j} \Omega}\right) . \\
\cdot \boldsymbol{P}\left(\mathrm{e}^{\mathrm{j} \Omega}\right) \boldsymbol{Q}^{\mathrm{H}}\left(\mathrm{e}^{\mathrm{j} \Omega}\right) .
\end{gathered}
$$

Therefore, the resulting eigenvalues on the diagonal of $\boldsymbol{P}\left(\mathrm{e}^{\mathrm{j} \Omega}\right) \boldsymbol{\Lambda}\left(\mathrm{e}^{\mathrm{j} \Omega}\right) \boldsymbol{P}^{\mathrm{H}}\left(\mathrm{e}^{\mathrm{j} \Omega}\right)$ and eigenvectors in the columns of $\boldsymbol{Q}\left(\mathrm{e}^{\mathrm{j} \Omega}\right) \boldsymbol{P}^{\mathrm{H}}\left(\mathrm{e}^{\mathrm{j} \Omega}\right)$ can be discontinuous. The statement of Section III-C that $\Lambda\left(\mathrm{e}^{\mathrm{j} \Omega}\right)$ can be continuous or even analytic for an analytic $\boldsymbol{R}(z)$ implies that this permutation matrix is selected appropriately. $^{7}$

Based on the argument for at least continuous $Q\left(\mathrm{e}^{\mathrm{j} \Omega}\right)$ and $\boldsymbol{\Lambda}\left(\mathrm{e}^{\mathrm{j} \Omega}\right)$ made in Section III-B, we here assume that permutations are chosen such that eigenvalues are at least continuous on the unit circle, i.e. that permutations of eigenvalues can only occur at algebraic multiplicities of those same eigenvalues, and are applied such that $2 \pi$-periodicity of all functions in (19) is retained. In the following we therefore distinguish three cases as characterised by the examples in Fig. 1:

a) non-overlapping eigenvalues $\lambda_{m}\left(\mathrm{e}^{\mathrm{j} \Omega}\right)$, where all eigenvalues have algebraic multiplicity one for all frequencies $\Omega$, such as the PSDs shown in Fig. 1(a);

b) overlapping, maximally smooth eigenvalues, such as shown in Fig. 1(b); and

c) overlapping, spectrally majorised PSDs as shown in Fig. 1(c).

Note that cases (a) and (c) are spectrally majorised, while cases (a) and (b) will be seen to yield analytic eigenvalues for $\Omega \in$ $\mathbb{R}$. Note that not all eigenvalues in (c) are differentiable for every value of $\Omega$, but they will later shown to be Lipschitz continuous. In the rest of this paper we treat the cases of distinct and overlapping eigenvalues separately.

\section{CAse of Distinct Eigenvalues}

In the case of distinct, non-overlapping eigenvalues $\lambda_{m}\left(\mathrm{e}^{\mathrm{j} \Omega}\right)$, $m=1 \ldots M$, spectral majorisation in (2) holds with strict inequality for all $\Omega$. As a result, the power spectra of the eigenvalues are smooth and distinct and, as in the example of Fig. 1(a), do not intersect.

\footnotetext{
${ }^{7}$ Recall from Section III-B that a discontinuous function of frequency will not admit an absolutely convergent Laurent or power series.
} 


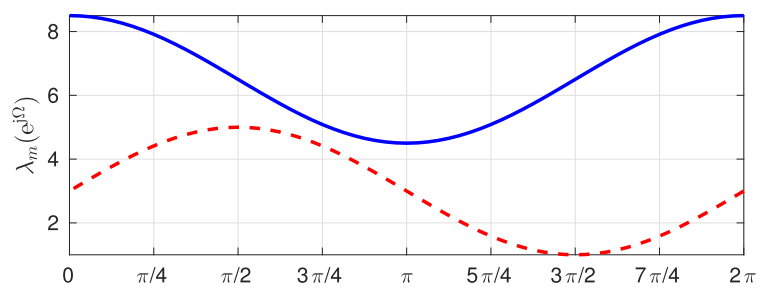

(a)

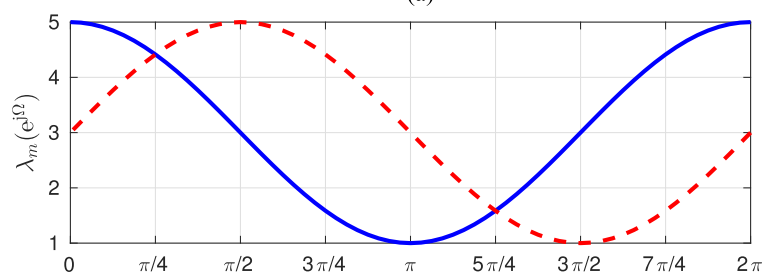

(b)

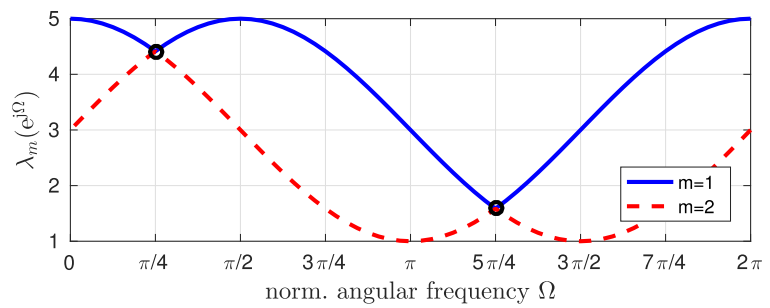

(c)

Fig. 1. Examples for (a) non-overlapping and overlapping eigenvalues with (b) smooth and (c) spectrally majorised PSDs. Non-differentiable points are indicated by black circles.

\section{A. Existence, Uniqueness and Approximation of Eigenvalues}

Theorem 1 (Existence and Uniqueness of Distinct Eigenvalues): Let $\boldsymbol{R}(z)$ be a parahermitian matrix which is analytic at least on an annulus containing the unit circle and whose EVD on the unit circle, as defined in (9), has distinct eigenvalues $\lambda_{m}\left(\mathrm{e}^{\mathrm{j} \Omega}\right), \forall \Omega$ and $m=1 \ldots M$. Then a matrix of eigenvalues of $\boldsymbol{R}(z)$ exists as a unique analytic Laurent series $\boldsymbol{\Gamma}(z)$ that matches $\boldsymbol{\Lambda}\left(\mathrm{e}^{\mathrm{j} \Omega}\right)=\operatorname{diag}\left\{\lambda_{1}\left(\mathrm{e}^{\mathrm{j} \Omega}\right) \ldots \lambda_{M}\left(\mathrm{e}^{\mathrm{j} \Omega}\right)\right\}$ on the unit circle.

Proof: If $\mathbf{R}(z)$ is analytic in the annulus $\rho<|z|<\rho^{-1}$ then we know from Section III-C that the eigenvalues $\lambda_{m}\left(\mathrm{e}^{\mathrm{j} \Omega}\right)$, $m=1, \ldots, M$ can be chosen to be analytic for real $\Omega$. Since analytic functions are Hölder continuous the discussion in Section III-B applies and therefore a potentially infinite order, matrix-valued Fourier series can be found that converges to $\boldsymbol{\Lambda}\left(\mathrm{e}^{\mathrm{j} \Omega}\right)$. Further since the eigenvalues are analytic on the unit circle, the Fourier series representation of the eigenvalues can be analytically continued to an annulus containing the unit circle via the substitution $z=\mathrm{e}^{\mathrm{j} \Omega}$. This gives the potentially infinite Laurent series $\boldsymbol{\Gamma}(z)$ representing the $M$ eigenvalues of $\boldsymbol{R}(z)$. This matrix of eigenvalues, $\boldsymbol{\Gamma}(z)$, matches $\boldsymbol{\Lambda}\left(\mathrm{e}^{\mathrm{j} \Omega}\right)$ on the unit circle, and therefore is unique as discussed.

In order to find an approximation of finite length to a Laurent or power series, consider that the Fourier series of the $m$ th eigenvalue takes the form $\lambda_{m}\left(\mathrm{e}^{\mathrm{j} \Omega}\right)=\lim _{N \longrightarrow \infty} \hat{\lambda}_{m}^{\langle N\rangle}\left(\mathrm{e}^{\mathrm{j} \Omega}\right)$, with

$$
\hat{\lambda}_{m}^{\langle N\rangle}\left(\mathrm{e}^{\mathrm{j} \Omega}\right)=\sum_{\ell=0}^{N} c_{m, \ell} \mathrm{e}^{\mathrm{j} \ell \Omega}+c_{m, \ell}^{*} \mathrm{e}^{-\mathrm{j} \ell \Omega}, \quad c_{m, \ell} \in \mathbb{C} .
$$

With $\hat{\boldsymbol{\Lambda}}^{\langle N\rangle}\left(\mathrm{e}^{\mathrm{j} \Omega}\right)=\operatorname{diag}\left\{\hat{\lambda}_{1}^{\langle N\rangle}\left(\mathrm{e}^{\mathrm{j} \Omega}\right), \ldots, \hat{\lambda}_{M}^{\langle N\rangle}\left(\mathrm{e}^{\mathrm{j} \Omega}\right)\right\}$, absolute convergence implies uniform convergence, such that for every $\epsilon_{\Lambda}>0$ there exists $N>0$ with

$$
\sup _{\Omega \in[0,2 \pi)}\left\|\hat{\boldsymbol{\Lambda}}^{\langle N\rangle}\left(\mathrm{e}^{\mathrm{j} \Omega}\right)-\boldsymbol{\Lambda}\left(\mathrm{e}^{\mathrm{j} \Omega}\right)\right\|<\epsilon_{\Lambda},
$$

where $\|\cdot\|$ is any matrix norm. As $N \rightarrow \infty, \epsilon_{\Lambda} \rightarrow 0$ at every frequency $\Omega$, so that the Fourier series (21) converges to $\Lambda\left(\mathrm{e}^{\mathrm{j} \Omega}\right)$. For finite $N$, an analytic continuation via the substitution $z=\mathrm{e}^{\mathrm{j} \Omega}$ into (20) is always possible, and yields a Laurent polynomial approximation $\hat{\Gamma}(z)$. Alternatively, a direct approximation of $\boldsymbol{\Lambda}\left(\mathrm{e}^{\mathrm{j} \Omega}\right)$ by Laurent polynomials is available via the Stone-Weierstrass theorem [41], [42], [47].

When approximating the exact eigenvalues $\Gamma(z)$ by Laurent polynomials of order $2 N$, a truncation error is incurred according to (21). Since the region of convergence of $\boldsymbol{\Gamma}(z)$ may be smaller than $\mathcal{D}$, we cannot make a statement here about how fast or slow such an approximation converges. The generally infinite-length nature of the Laurent series representation of the eigenvalues will be evident when we consider the "simple" case of a $2 \times 2$ parahermitian matrix next, followed by an example problem that was stated but not solved in [26].

\section{B. Eigenvalues of $2 \times 2$ Parahermitian Matrices}

In this section we exemplify the existence and uniqueness of the eigenvalues of an arbitrary parahermitian matrix $\boldsymbol{R}(z)$ : $\mathbb{C} \rightarrow \mathbb{C}^{2 \times 2}$. These eigenvalues $\gamma_{1,2}(z)$ can be directly computed in the $z$-domain as the roots of

$$
\operatorname{det}\{\gamma(z) \mathbf{I}-\boldsymbol{R}(z)\}=\gamma^{2}(z)-T(z) \gamma(z)+D(z)=0
$$

with determinant $D(z)=\operatorname{det}\{\boldsymbol{R}(z)\}$ and trace $T(z)=$ trace $\{\boldsymbol{R}(z)\}$. This leads to

$$
\gamma_{1,2}(z)=\frac{1}{2} T(z) \pm \frac{1}{2} \sqrt{T(z) T^{\mathrm{P}}(z)-4 D(z)} .
$$

The argument under the square root is parahermitian and can be factored into $Y(z) Y^{\mathrm{P}}(z)=T(z) T^{\mathrm{P}}(z)-4 D(z)$, where $Y(z)$ has all zeros and poles inside the unit circle, and $Y^{\mathrm{P}}(z)$ has all zeros and poles outside the unit circle. In the rare case that $Y(z)$ has no poles and all zeros have multiplicity $2 N, N \in \mathbb{N}$, the solution for (22) is a Laurent polynomial. If both poles and zeros of $Y$ have multiplicity $2 N, N \in \mathbb{N}$, the eigenvalues are rational functions in $z$.

In general, the square root in (22) will be neither polynomial nor rational, as recognised for a Laurent polynomial QR decomposition in [27]. Within the convergence region $|z|>\rho$, where $\rho<1$ is the maximum modulus of all poles and zeros of $Y(z)$, we take the square root of each zero $\beta$ and pole $\alpha$ in $Y(z)$ 




Fig. 2. MacLaurin series expansion coefficients for square root of a zero or pole.

separately. Then a Maclaurin series expansion gives

$$
\begin{aligned}
\sqrt{1-\beta z^{-1}} & =\sum_{n=0}^{\infty} \xi_{n} \beta^{n} z^{-n} \\
\frac{1}{\sqrt{1-\alpha z^{-1}}} & =\left(\sum_{n=0}^{\infty} \xi_{n} \alpha^{n} z^{-n}\right)^{-1} \\
& =\sum_{n=0}^{\infty} \chi_{n} \alpha^{n} z^{-n}
\end{aligned}
$$

with

$$
\begin{aligned}
& \xi_{n}=(-1)^{n}\left(\begin{array}{c}
\frac{1}{2} \\
n
\end{array}\right)=\frac{(-1)^{n}}{n !} \prod_{i=0}^{n-1}\left(\frac{1}{2}-i\right), \\
& \chi_{n}=(-1)^{n}\left(\begin{array}{c}
-\frac{1}{2} \\
n
\end{array}\right)=\frac{(-1)^{n-1}}{n !} \prod_{i=0}^{n-1}\left(\frac{1}{2}+i\right) .
\end{aligned}
$$

The MacLaurin coefficients $\xi_{n}$ and $\chi_{n}$ for $n=0 \ldots 50$ are shown in Fig. 2.

Thus, a stable causal square root $Y(z)^{1 / 2}$ is obtained. The square root of $Y^{\mathrm{P}}(z)$ with a convergence region $|z|<\rho^{-1}$ is given by $\left(Y^{\mathrm{P}}(z)\right)^{1 / 2}=\left(Y(z)^{1 / 2}\right)^{P}$. The representation of the square root is therefore complete, and can be accomplished by an infinite order rational function in $z$ via (23) and (24), or by a Laurent series via (23) and (25). The eigenvalues in (22) therefore exist as convergent but generally infinite Laurent series [41] but clearly could be approximated by finite order rational functions or Laurent polynomials.

Example. To demonstrate the calculation of eigenvalues, we consider the parahermitian matrix

$$
\boldsymbol{R}(z)=\left[\begin{array}{cc}
1 & 1 \\
1 & -2 z+6-2 z^{-1}
\end{array}\right]
$$

stated in [26], which has poles at $z=0$ and $z \rightarrow \infty$ but is analytic in $\{z: z \in \mathbb{C}, z \neq 0, \infty\}$.

Using (23) and (25), the approximate Laurent polynomial eigenvalues are characterised in Fig. 3 in terms of their PSDs $\hat{\gamma}_{m}\left(\mathrm{e}^{\mathrm{j} \Omega}\right)$, expansion coefficients $\hat{\gamma}_{m}[\tau]$ such that $\hat{\gamma}_{m}(z)=$ $\sum_{\tau} \hat{\gamma}_{m}[\tau] z^{-\tau}$, and their log-moduli. The latter in Fig. 3(c) shows the rapid decay of the Laurent series, justifying a Laurent polynomial approximation.

This expands on the result in [26], where it was shown that $\boldsymbol{R}(z)$ in (26) does not have polynomial eigenvalues, but where

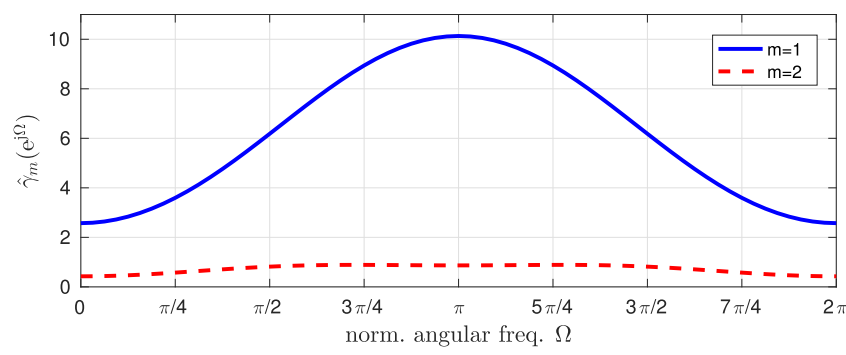

(a)

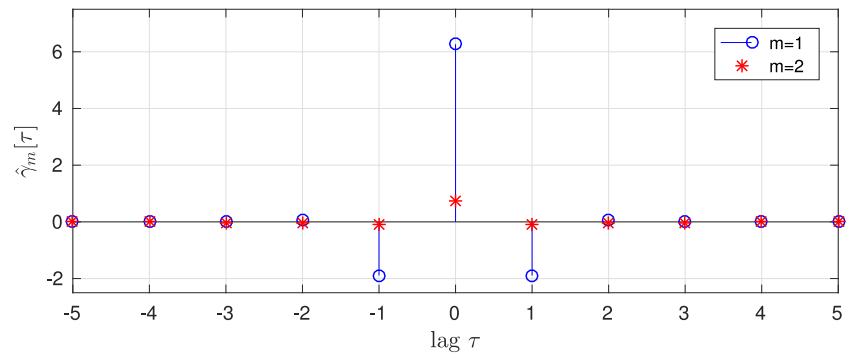

(b)

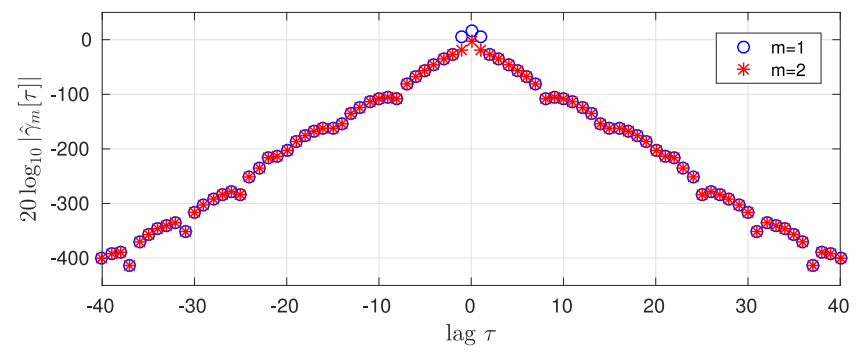

(c)

Fig. 3. Approximate eigenvalues of $\mathbf{R}(z)$ in (26). (a) Power spectral densities. (b) Laurent polynomial coefficients. (c) Decay of power series.

no polynomial or rational approximation was given. The example demonstrates that an approximate solution using Laurent polynomials exists, which can be arbitrarily accurate for a sufficiently high order of $\hat{\gamma}_{1,2}(z)$, as supported by Theorem 1 .

\section{Existence, Ambiguity and Approximation of Eigenvectors}

Recall that the eigenvalues of $\boldsymbol{R}\left(\mathrm{e}^{\mathrm{j} \Omega}\right)$ are assumed to possess non-overlapping PSDs, i.e the eigenvalues for all frequencies $\Omega$ have algebraic multiplicity one, i.e. $C=1$. The subspaces in Section III-D can now all be treated as one-dimensional, and eigenvectors are therefore uniquely identified, save for the phase shift in (3). Since this phase shift is arbitrary at every frequency $\Omega$, the polynomial eigenvectors are defined up to an arbitrary phase response. With this, some of the expressions in Section III-D simplify, and permit the statement of the following theorem.

Theorem 2 (Existence and ambiguity of eigenvectors for distinct eigenvalues): Let $\boldsymbol{R}(z): \mathbb{C} \rightarrow \mathbb{C}^{M \times M}$ be a parahermitian matrix whose EVD on the unit circle, as defined in (9), has distinct eigenvalues $\lambda_{m}\left(\mathrm{e}^{\mathrm{j} \Omega}\right), \forall \Omega$ and $m=1 \ldots M$. Each corresponding eigenvector $\mathbf{q}_{m}\left(\mathrm{e}^{\mathrm{j} \Omega}\right)$ can have an arbitrary phase response. Then for any phase response that creates a Hölder continuous $\mathbf{q}_{m}\left(\mathrm{e}^{\mathrm{j} \Omega}\right)$, an absolutely convergent vector-valued series $\boldsymbol{u}_{m}(z)$ exists which matches $\mathbf{q}_{m}\left(\mathrm{e}^{\mathrm{j} \Omega}\right)$ on the unit circle. 
Proof: Considering the $m$ th eigenvalue and -vector, $\lambda_{m}\left(\mathrm{e}^{\mathrm{j} \Omega}\right)$ and $\boldsymbol{q}_{m}\left(\mathrm{e}^{\mathrm{j} \Omega}\right)$, the spectral distance from its nearest neighbour at frequency $\Omega$ is [31]

$$
\delta_{m}\left(\mathrm{e}^{\mathrm{j} \Omega}\right)=\min _{n \neq m}\left|\lambda_{n}\left(\mathrm{e}^{\mathrm{j} \Omega}\right)-\lambda_{m}\left(\mathrm{e}^{\mathrm{j} \Omega}\right)\right|>0 .
$$

Now, in (16), $\boldsymbol{E}_{21}\left(\mathrm{e}^{\mathrm{j} \Omega}\right): \mathbb{R} \rightarrow \mathbb{C}^{M-1}$ is a vector, and if (17) holds, then (18) simplifies to

$\operatorname{dist}\left\{\boldsymbol{q}_{m}\left(\mathrm{e}^{\mathrm{j} \Omega}\right), \boldsymbol{q}_{m}\left(\mathrm{e}^{\mathrm{j}(\Omega+\Delta \Omega)}\right)\right\} \leq \frac{4}{\delta_{m}\left(\mathrm{e}^{\mathrm{j} \Omega}\right)}\left\|\mathbf{E}_{21}\left(\mathrm{e}^{\mathrm{j} \Omega}, \Delta \Omega\right)\right\|_{\mathrm{F}}$.

As $\Delta \Omega \longrightarrow 0$, also $\operatorname{dist}\left\{\boldsymbol{q}_{m}\left(\mathrm{e}^{\mathrm{j} \Omega}\right), \boldsymbol{q}_{m}\left(\mathrm{e}^{\mathrm{j}(\Omega+\Delta \Omega)}\right)\right\} \longrightarrow 0$, and the one-dimensional subspace within which each eigenvector resides must evolve continuously with frequency. It can be further shown that the eigenvectors can be chosen to be analytic [35].

Because of the phase ambiguity in (3), each eigenvector can be given an arbitrary phase response $\Phi_{m}\left(\mathrm{e}^{\mathrm{j} \Omega}\right)$, with $\left|\Phi_{m}\left(\mathrm{e}^{\mathrm{j} \Omega}\right)\right|=1 \forall \Omega \in[0 ; 2 \pi), m=1 \ldots M$ without affecting the orthonormality of eigenvectors. Only if $\Phi_{m}\left(\mathrm{e}^{\mathrm{j} \Omega}\right)$ is selected such that the $M$ elements of $\boldsymbol{q}_{m}\left(\mathrm{e}^{\mathrm{j} \Omega}\right)$ vary Hölder-continuously in $\Omega$, then analogously to the proof of Theorem 1, a Höldercontinuous $\boldsymbol{q}_{m}\left(\mathrm{e}^{\mathrm{j} \Omega}\right)$ has an absolutely convergent Fourier series [40]

$$
\hat{\boldsymbol{q}}_{m}^{\langle N\rangle}\left(\mathrm{e}^{\mathrm{j} \Omega}\right)=\sum_{\ell=-N}^{N} \boldsymbol{d}_{m, \ell} \cdot \mathrm{e}^{\mathrm{j} \ell \Omega},
$$

where $\boldsymbol{d}_{m, \ell} \in \mathbb{C}^{M}$ and $\left\|\hat{\boldsymbol{q}}_{m}^{\langle N\rangle}\left(\mathrm{e}^{\mathrm{j} \Omega}\right)-\boldsymbol{q}_{m}\left(\mathrm{e}^{\mathrm{j} \Omega}\right)\right\| \longrightarrow 0 \forall \Omega$ as $N \longrightarrow \infty$. According to Section III-B, this admits an absolutely convergent power or Laurent series $\boldsymbol{u}_{m}(z)$. If additionally the phase response does not just create a $\boldsymbol{q}_{m}\left(\mathrm{e}^{\mathrm{j} \Omega}\right)$ that is Hölder continuous but one that is also analytic in $\Omega$, then the continuation to an analytic $\boldsymbol{u}_{m}(z)$ exists.

The selection of the phase response does not just cause ambiguity of the eigenvectors, but also affects the properties of a Laurent polynomial approximation of these eigenvectors. An appropriate phase response may e.g. admit a causal, polynomial approximation. Further, we distinguish below between the selection of a continuous and a discontinuous phase response, leading to matrices $\boldsymbol{Q}\left(\mathrm{e}^{\mathrm{j} \Omega}\right)$ that are continuous and discontinuous in $\Omega$, respectively:

- Hölder Continuous Case. This case is covered by Theorem 2, which requires phase responses that are otherwise arbitrary but constrained for $\boldsymbol{q}_{m}\left(\mathrm{e}^{\mathrm{j} \Omega}\right), m=1 \ldots M$, to be Hölder continuous for eigenvectors $\boldsymbol{U}(z)$ to exist as convergent Laurent or power series. Ambiguity w.r.t. the phase response implies that for any differently selected continuous phase response, a different $\boldsymbol{U}(z)$ emerges. Approximations of $\boldsymbol{U}(z)$ by Laurent polynomials $\hat{\boldsymbol{U}}(z)$ can be obtained by truncation; this approximation will improve with the approximation order and smoothness of the phase response. A special case arises if the phase responses are selected such that $\boldsymbol{Q}\left(\mathrm{e}^{\mathrm{j} \Omega}\right)$ is analytic, which directly implies a convergent power series $\boldsymbol{U}(z)$.

- Discontinuous Case. If $\boldsymbol{q}_{m}\left(\mathrm{e}^{\mathrm{j} \Omega}\right)$ is piecewise continuous and possesses a discontinuity at $\Omega=\Omega_{0}$, then there does not exist a convergent Laurent or power series representation of the eigenvector. However since $\boldsymbol{q}_{m}\left(\mathrm{e}^{\mathrm{j} \Omega}\right)$ is periodic in $\Omega$, an at least pointwise convergent Fourier series does exist, and at the point $\Omega_{0}$ will converge to

$$
\begin{aligned}
\lim _{N \rightarrow \infty} \hat{\boldsymbol{q}}_{m}^{\langle N\rangle}\left(\mathrm{e}^{\mathrm{j} \Omega_{0}}\right)=\frac{1}{2} \lim _{\Omega \rightarrow 0} & \left(\boldsymbol{q}_{m}\left(\mathrm{e}^{\mathrm{j}\left(\Omega_{0}-\Omega\right)}\right)+\right. \\
& \left.+\boldsymbol{q}_{m}\left(\mathrm{e}^{\mathrm{j}\left(\Omega_{0}+\Omega\right)}\right)\right) .
\end{aligned}
$$

Since (28) is the mean value between the left- and right function values at the discontinuity, a Fourier series representation will not match $\boldsymbol{q}_{m}\left(\mathrm{e}^{\mathrm{j} \Omega}\right)$ at least at $\Omega_{0}$. An approximation by a Laurent polynomial $\hat{\boldsymbol{U}}(z)$ of sufficiently high order, evaluated on the unit circle, will converge to the mean values of $\boldsymbol{Q}\left(\mathrm{e}^{\mathrm{j} \Omega}\right)$ according to (28) at the discontinuities, and Gibbs phenomena may occur in the proximity. For the case where eigenvalues $\boldsymbol{q}_{m}\left(\mathrm{e}^{\mathrm{j} \Omega}\right)$ are neither Höldercontinuous nor discontinuous, uniform convergence of the Fourier series cannot be guaranteed [40]; this case is outwith the scope of this paper, but we refer the interested reader to e.g. [40] for the appropriate conditions on convergence.

\section{Case of Eigenvalues With Multiplicities}

Following the consideration of distinct, non-overlapping eigenvalues $\lambda_{m}\left(\mathrm{e}^{\mathrm{j} \Omega}\right), m=1 \ldots M$, in Section IV, we now address the case where the PSDs of eigenvalues intersect or touch, i.e. there is an algebraic multiplicity of eigenvalues greater than one at one or more frequencies. Because of an ambiguity of how to associate eigenvalues across the frequency spectrum, similar to the permutation problem in broadband blind source separation, a distinction is made between maximally smooth and spectrally majorised PSDs, as illustrated by the examples in Fig. 1(b) and (c), respectively.

\section{A. Existence, Uniqueness and Approximation of Eigenvalues}

Section III-C indicated that eigenvalues of $\boldsymbol{R}\left(\mathrm{e}^{\mathrm{j} \Omega}\right)$, that have an algebraic multiplicity of one, can be chosen to be analytic (hence continuous and infinitely differentiable) functions on the unit circle [35]. Therefore if we constrain the eigenvalues to be continuous, then $\Lambda\left(\mathrm{e}^{\mathrm{j} \Omega}\right)$ has to be at the very least piecewise analytic on the unit circle.

It follows that if any two eigenvalues $\lambda_{m}\left(\mathrm{e}^{\mathrm{j} \Omega}\right)$ and $\lambda_{n}\left(\mathrm{e}^{\mathrm{j} \Omega}\right)$, $m, n=1 \ldots M$, are permuted at an algebraic multiplicity greater than one, then

$$
\sup _{\Omega_{1}, \Omega_{2} \in \mathbb{R}}\left|\lambda_{m}\left(\mathrm{e}^{\mathrm{j} \Omega_{1}}\right)-\lambda_{n}\left(\mathrm{e}^{\mathrm{j} \Omega_{2}}\right)\right| \leq L\left|\mathrm{e}^{\mathrm{j} \Omega_{1}}-\mathrm{e}^{\mathrm{j} \Omega_{2}}\right|
$$

holds with $m, n=1 \ldots M$, the Lipschitz constant

$$
L=\max _{\substack{m \in\{1,2, \ldots M\} \\ \Omega \in \mathbb{R} \backslash \mathcal{M}}}\left|\frac{\mathrm{d}}{\mathrm{d} \Omega} \lambda_{m}\left(\mathrm{e}^{\mathrm{j} \Omega}\right)\right|,
$$

and $\mathcal{M}$ the set of frequency points where eigenvalues have an algebraic multiplicity greater than one. In between these points, the r.h.s. of (29) exists because the eigenvalues are piecewise analytic. Therefore any permutation of eigenvalues is Lipschitz continuous, which matches with (10) for $\alpha=1$ and $L=C$. 


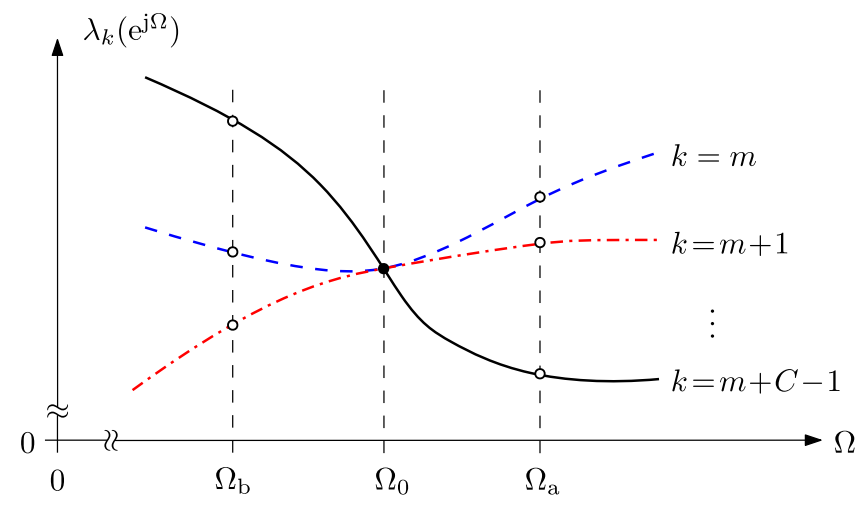

Fig. 4. Cluster of $C$ eigenvalues in the neighbourhood of a $C$-fold multiplicity at $\Omega=\Omega_{0}$.

Hence, this is a stronger condition than Hölder continuity, and therefore guarantees the representation by an absolutely convergent Fourier series in analogy to the arguments in Section IV-A; an alternative representation in terms of Laurent series can be reached via the Stone-Weierstrass theorem. This leads to the following theorem:

Theorem 3 (Existence and uniqueness of eigenvalues of a parahermitian matrix EVD): Let $\boldsymbol{R}(z)$ be an analytic parahermitian matrix whose EVD on the unit circle, as defined in (9), has an eigenvalue matrix $\Lambda\left(\mathrm{e}^{\mathrm{j} \Omega}\right), \forall \Omega \in \mathbb{R}$. Then the matrix of eigenvalues $\Gamma(z)$ exists as an absolutely convergent Laurent series. Uniqueness requires additional constraints on the permutation of eigenvalues on the unit circle, such as maximal smoothness or spectral majorisation, with consequences for the order of a Laurent polynomial approximation $\hat{\boldsymbol{\Gamma}}(z)$ of $\boldsymbol{\Gamma}(z)$.

Proof: This is covered by Theorem 1 for distinct eigenvalues, and otherwise follows from the above reasoning.

The approximation of eigenvalues by Laurent polynomials, here argued in terms of a truncated Fourier series expansion (see Theorem 1), is guaranteed to be analytic because of the restriction to a finite order. However, differences in the convergence speed can be noted: we expect faster convergence for analytic, i.e. maximally smooth eigenvalues than for spectrally majorised ones, since for the latter $\Lambda\left(\mathrm{e}^{\mathrm{j} \Omega}\right)$ is only piecewise analytic on the unit circle. Therefore generally higher order Laurent polynomials are required when approximating spectrally majorised eigenvalues as compared to the maximally smooth case, if eigenvalues have an algebraic multiplicity greater than one on the unit circle. This outcome of Theorem 3 agrees with results in [9], as well as with experimental findings in [48] based on factorisations for different source models - with both distinct and spectrally majorised sources—of a space-time covariance matrix.

\section{B. Uniqueness and Ambiguity of Eigenvectors}

We now inspect the eigenvectors in the vicinity of a $C$-fold algebraic multiplicity of eigenvalues at $\Omega=\Omega_{0}$, as shown in Fig. 4. By assumption $\boldsymbol{R}\left(\mathrm{e}^{\mathrm{j} \Omega}\right)$ is analytic and, from Section III-C, $\boldsymbol{\Lambda}\left(\mathrm{e}^{\mathrm{j} \Omega}\right)$ can be chosen to be analytic for all $\Omega$, including $\Omega_{0}$. In this case, Rellich [35] shows that the eigenvectors can be analytic. We want to explore the behaviour of $\boldsymbol{Q}\left(\mathrm{e}^{\mathrm{j} \Omega}\right)$, and particularly the conditions under which it has a unique analytic solution. That this is the case seems to be understood in various texts [30], [35], [45] but the authors have not found a definitive reference. Hence we offer Lemma 1, below, as an alternative proof.

In rare cases we may find identical eigenvalues. Two eigenvalues $\lambda_{m}\left(\mathrm{e}^{\mathrm{j} \Omega}\right)$ and $\lambda_{\ell}\left(\mathrm{e}^{\mathrm{j} \Omega}\right)$ are identical if $\lambda_{m}\left(\mathrm{e}^{\mathrm{j} \Omega}\right)=$ $\lambda_{\ell}\left(\mathrm{e}^{\mathrm{j} \Omega}\right) \forall \Omega$. In the following we exclude this case; an ambiguity is expected from (4), but the presence of identical eigenvalues makes the analysis more involved and the case is usually avoided by estimation and rounding errors in $\boldsymbol{R}(z)$.

Lemma 1 (Existence and uniqueness of analytic eigenspaces on the unit circle): Under the assumptions of Theorem 3 and in the absence of identical eigenvalues, there exist unique 1-d subspaces for analytic eigenvectors in $\boldsymbol{Q}\left(\mathrm{e}^{\mathrm{j} \Omega}\right)$ if and only if eigenvalues in $\Lambda\left(\mathrm{e}^{\mathrm{j} \Omega}\right)$ are selected to be analytic across algebraic multiplicities.

Proof: That it is possible to choose analytic eigenvectors when the eigenvalues are all chosen to be analytic follows from Rellich [35]. To see the 'only if' part, we now assume that the eigenvectors are chosen to be analytic, and show that this can only occur if the eigenvalues are also analytic.

By exploiting Theorem 1 between multiplicities, we know that continuous eigenvalues have to be at the very least piecewise analytic on the unit circle. Further, between the points of multiplicity greater than one, these functions are unique (up to the order they appear in the matrix $\Lambda\left(\mathrm{e}^{\mathrm{j} \Omega}\right)$ ). If the analytic eigenvalues from Rellich are $\Lambda_{0}\left(\mathrm{e}^{\mathrm{j} \Omega}\right)$, then the only alternative choice for the eigenvalue matrix is

$$
\boldsymbol{\Lambda}\left(\mathrm{e}^{\mathrm{j} \Omega}\right)= \begin{cases}\boldsymbol{\Lambda}_{\mathrm{b}}\left(\mathrm{e}^{\mathrm{j} \Omega}\right)=\mathbf{P}_{\mathrm{b}} \boldsymbol{\Lambda}_{0}\left(\mathrm{e}^{\mathrm{j} \Omega}\right) \mathbf{P}_{\mathrm{b}}^{\mathrm{H}}, & \Omega \leq \Omega_{0}, \\ \boldsymbol{\Lambda}_{\mathrm{a}}\left(\mathrm{e}^{\mathrm{j} \Omega}\right)=\mathbf{P}_{\mathrm{a}} \boldsymbol{\Lambda}_{0}\left(\mathrm{e}^{\mathrm{j} \Omega}\right) \mathbf{P}_{\mathrm{a}}^{\mathrm{H}}, & \Omega \geq \Omega_{0},\end{cases}
$$

where subscripts 'a' and ' $b$ ' indicate 'above' and 'below' $\Omega_{0}$, and $\mathbf{P}_{\mathrm{a}}, \mathbf{P}_{\mathrm{b}} \in \mathbb{R}^{M \times M}$ are permutation matrices. Because we can arbitrarily order the eigenvalues in $\Lambda_{0}\left(\mathrm{e}^{\mathrm{j} \Omega}\right)$ and their corresponding eigenvectors in $\boldsymbol{Q}\left(\mathrm{e}^{\mathrm{j} \Omega}\right)$ without affecting their analyticity, w.l.o.g. we set $\boldsymbol{\Lambda}_{\mathrm{a}}\left(\mathrm{e}^{\mathrm{j} \Omega}\right)=\boldsymbol{\Lambda}_{0}\left(\mathrm{e}^{\mathrm{j} \Omega}\right)$, i.e. $\mathbf{P}_{\mathrm{a}}=\mathbf{I}$.

With reference to (19), we have

$$
\boldsymbol{R}\left(\mathrm{e}^{\mathrm{j} \Omega}\right)= \begin{cases}\boldsymbol{Q}\left(\mathrm{e}^{\mathrm{j} \Omega}\right) \boldsymbol{\Lambda}_{\mathrm{b}}\left(\mathrm{e}^{\mathrm{j} \Omega}\right) \boldsymbol{Q}^{\mathrm{H}}\left(\mathrm{e}^{\mathrm{j} \Omega}\right), & \Omega \leq \Omega_{0}, \\ \boldsymbol{Q}\left(\mathrm{e}^{\mathrm{j} \Omega}\right) \boldsymbol{\Lambda}_{\mathrm{a}}\left(\mathrm{e}^{\mathrm{j} \Omega}\right) \boldsymbol{Q}^{\mathrm{H}}\left(\mathrm{e}^{\mathrm{j} \Omega}\right), & \Omega>\Omega_{0},\end{cases}
$$

where $\boldsymbol{Q}\left(\mathrm{e}^{\mathrm{j} \Omega}\right)$ is assumed to be analytic, and $\boldsymbol{R}\left(\mathrm{e}^{\mathrm{j} \Omega}\right)$ is analytic by premise. With this, we can define $n$th order derivatives approaching $\Omega_{0}$ from above and below,

$$
\begin{aligned}
& \lim _{\mathrm{a}} \longrightarrow \Omega_{0}+ \frac{\mathrm{d}^{n}}{\mathrm{~d} \Omega_{\mathrm{a}}^{n}} \boldsymbol{R}\left(\mathrm{e}^{\mathrm{j} \Omega_{\mathrm{a}}}\right)=\mathbf{R}_{\mathrm{a}}^{(n)}, \\
& \lim _{\Omega_{\mathrm{b}} \longrightarrow \Omega_{0}-} \frac{\mathrm{d}^{n}}{\mathrm{~d} \Omega_{\mathrm{b}}^{n}} \boldsymbol{R}\left(\mathrm{e}^{\mathrm{j} \Omega_{\mathrm{b}}}\right)=\mathbf{R}_{\mathrm{b}}^{(n)},
\end{aligned}
$$

and state $\mathbf{R}_{\mathrm{a}}^{(n)}=\mathbf{R}_{\mathrm{b}}^{(n)} \forall n \in \mathbb{N}$. From (30) it is clear that we can define quantities $\boldsymbol{\Lambda}_{\mathrm{a}}^{(n)}$ and $\boldsymbol{\Lambda}_{\mathrm{b}}^{(n)}$ in analogy to (32) and (33). In order to investigate if there is ambiguity in the choice of eigenvectors, and to see how eigenspaces behave in the vicinity of $\Omega_{0}$, we define derivatives $\mathbf{Q}_{\mathrm{a}}^{(n)}$ and $\mathbf{Q}_{\mathrm{b}}^{(n)}$ of $\boldsymbol{Q}\left(\mathrm{e}^{\mathrm{j} \Omega}\right)$ from 
above and below $\Omega_{0}$ analogously to (32) and (33). Note that because of the analyticity of $\boldsymbol{Q}\left(\mathrm{e}^{\mathrm{j} \Omega}\right), \mathbf{Q}_{\mathrm{a}}^{(n)}=\mathbf{Q}_{\mathrm{b}}^{(n)} \forall n \in \mathbb{N}$.

For $\mathbf{R}_{\mathrm{a}}^{(0)}=\mathbf{R}_{\mathrm{b}}^{(0)}$, we take the EVD on either side, and with $\mathbf{Q}_{\mathrm{a}}^{(0)}=\mathbf{Q}_{\mathrm{b}}^{(0)}$ and the premise of continuous eigenvalues, i.e. $\boldsymbol{\Lambda}_{\mathrm{a}}^{(0)}=\mathbf{P}_{\mathrm{b}}^{\mathrm{H}} \boldsymbol{\Lambda}_{\mathrm{b}}^{(0)} \mathbf{P}_{\mathrm{b}}$ due to (30), obtain

$$
\boldsymbol{Q}_{\mathrm{b}}^{(0)} \mathbf{P}_{\mathrm{b}}^{\mathrm{H}} \boldsymbol{\Lambda}_{\mathrm{b}}^{(0)} \mathbf{P}_{\mathrm{b}} \boldsymbol{Q}_{\mathrm{b}}^{(0), \mathrm{H}}=\boldsymbol{Q}_{\mathrm{b}}^{(0)} \boldsymbol{\Lambda}_{\mathrm{b}}^{(0)} \boldsymbol{Q}_{\mathrm{b}}^{(0), \mathrm{H}}
$$

or

$$
\mathbf{P}_{\mathrm{b}}^{\mathrm{H}} \boldsymbol{\Lambda}_{\mathrm{b}}^{(0)} \mathbf{P}_{\mathrm{b}}-\boldsymbol{\Lambda}_{\mathrm{b}}^{(0)}=\mathbf{0} .
$$

For the first derivative $\mathbf{R}_{\mathrm{a}}^{(1)}$, the product rule can be applied to the EVD factorisation,

$$
\mathbf{R}_{\mathrm{a}}^{(1)}=\mathbf{Q}_{\mathrm{a}}^{(1)} \boldsymbol{\Lambda}_{\mathrm{a}}^{(0)} \mathbf{Q}_{\mathrm{a}}^{(0)}+\mathbf{Q}_{\mathrm{a}}^{(0)} \boldsymbol{\Lambda}_{\mathrm{a}}^{(1)} \mathbf{Q}_{\mathrm{a}}^{(0)}+\mathbf{Q}_{\mathrm{a}}^{(0)} \boldsymbol{\Lambda}_{\mathrm{a}}^{(0)} \mathbf{Q}_{\mathrm{a}}^{(1)} \text {. }
$$

Taking the derivative of the r.h.s. of (34) and using a similar expression for $\mathbf{R}_{\mathrm{b}}^{(1)}$, and equating $\mathbf{R}_{\mathrm{a}}^{(1)}=\mathbf{R}_{\mathrm{b}}^{(1)}$, we find that

$$
\begin{aligned}
\mathbf{Q}_{\mathrm{b}}^{(1)}\left(\mathbf{P}_{\mathrm{b}}^{\mathrm{H}} \boldsymbol{\Lambda}_{\mathrm{b}}^{(0)} \mathbf{P}_{\mathrm{b}}-\boldsymbol{\Lambda}_{\mathrm{b}}^{(0)}\right) \mathbf{Q}_{\mathrm{b}}^{(0), \mathrm{H}}+ \\
+\mathbf{Q}_{\mathrm{b}}^{(0)}\left(\mathbf{P}_{\mathrm{b}}^{\mathrm{H}} \boldsymbol{\Lambda}_{\mathrm{b}}^{(0)} \mathbf{P}_{\mathrm{b}}-\boldsymbol{\Lambda}_{\mathrm{b}}^{(0)}\right) \mathbf{Q}_{\mathrm{b}}^{(1), \mathrm{H}}+ \\
\quad+\mathbf{Q}_{\mathrm{b}}^{(0)}\left(\mathbf{P}_{\mathrm{b}}^{\mathrm{H}} \boldsymbol{\Lambda}_{\mathrm{b}}^{(1)} \mathbf{P}_{\mathrm{b}}-\boldsymbol{\Lambda}_{\mathrm{b}}^{(1)}\right) \boldsymbol{Q}_{\mathrm{b}}^{(0), \mathrm{H}}=\mathbf{0} .
\end{aligned}
$$

Because of (35), the first two terms are zero, and we obtain $\mathbf{P}_{\mathrm{b}}^{\mathrm{H}} \boldsymbol{\Lambda}_{\mathrm{b}}^{(1)} \mathbf{P}_{\mathrm{b}}-\boldsymbol{\Lambda}_{\mathrm{b}}^{(1)}=\mathbf{0}$. By induction it can be shown that for $\mathbf{R}_{\mathrm{a}}^{(n)}=\mathbf{R}_{\mathrm{b}}^{(n)}$ indeed $\mathbf{P}_{\mathrm{b}}^{\mathrm{H}} \boldsymbol{\Lambda}_{\mathrm{b}}^{(n)} \mathbf{P}_{\mathrm{b}}-\boldsymbol{\Lambda}_{\mathrm{b}}^{(n)}=\mathbf{0} \forall n \in \mathbb{N}$, or

$$
\boldsymbol{\Lambda}_{\mathrm{b}}^{(n)} \mathbf{P}_{\mathrm{b}}-\mathbf{P}_{\mathrm{b}} \boldsymbol{\Lambda}_{\mathrm{b}}^{(n)}=\mathbf{0} \forall n \in \mathbb{N} .
$$

If $p_{m, \ell}$ is the element in the $m$ th row and $\ell$ th column of $\mathbf{P}_{\mathrm{b}}$, then elementwise, (36) demands

$$
p_{m, \ell}\left(\lambda_{\mathrm{b}, \ell}^{(n)}-\lambda_{\mathrm{b}, m}^{(n)}\right)=0 \forall n \in \mathbb{N},
$$

with $\lambda_{\mathrm{b}, m}^{(n)}$ the $m$ th diagonal entry of $\boldsymbol{\Lambda}_{\mathrm{b}}^{(n)}$.

In the absence of identical eigenvalues, even if the $\ell$ th and $m$ th eigenvalues, $m, \ell \in\{1 \ldots M\}, m \neq \ell$, belong to the cluster forming a $C$-fold algebraic multiplicity at $\Omega_{0}$, they will differ in at least one differentiation $n$, and hence $p_{m, \ell}=0$. As an example, in Fig. 4, the 0th and 1st order derivatives of $\lambda_{m}\left(\mathrm{e}^{\mathrm{j} \Omega}\right)$ and $\lambda_{m+1}\left(\mathrm{e}^{\mathrm{j} \Omega}\right)$ match at $\Omega=\Omega_{0}$, but the $n=2$ nd order derivatives differ. Therefore, $\mathbf{P}_{\mathrm{b}}$ must be a diagonal matrix. Further, unitarity, and the fact that $\mathbf{P}_{\mathrm{b}}$ is a permutation matrix enforces the constraint $p_{m, m}=1, m=1 \ldots M$ i.e. $\mathbf{P}_{\mathrm{b}}=\mathbf{I}$. Thus from (30), recalling that $\mathbf{P}_{\mathrm{a}}=\mathbf{I}$, we must have $\boldsymbol{\Lambda}\left(\mathrm{e}^{\mathrm{j} \Omega}\right)=\boldsymbol{\Lambda}_{0}\left(\mathrm{e}^{\mathrm{j} \Omega}\right)$. Therefore analytic eigenvectors are possible if and only if eigenvalues are analytically continued across $\Omega_{0}$.

Recall that the eigenvectors in $\boldsymbol{Q}\left(\mathrm{e}^{\mathrm{j} \Omega}\right)$ can possess arbitrary phase responses; as long as the latter are analytic, $\boldsymbol{Q}\left(\mathrm{e}^{\mathrm{j} \Omega}\right)$ will remain analytic. While this permits some ambiguity, under the exclusion of identical eigenvalues, each eigenvector must however be orthogonal to the remaining eigenvectors, and hence there exist unique 1-d subspaces within which analytic eigenvectors reside.

Analyticity or at least Hölder continuity of $\boldsymbol{Q}\left(\mathrm{e}^{\mathrm{j} \Omega}\right)$ requires that $\boldsymbol{\Lambda}\left(\mathrm{e}^{\mathrm{j} \Omega}\right)$ is analytic, and that the arbitrary phase response of $\boldsymbol{Q}\left(\mathrm{e}^{\mathrm{j} \Omega}\right)$ is selected analytic or at least Hölder continuous.
We focus next on extending the eigenvalues and eigenvectors to functions in $z$.

Theorem 4 (Existence and ambiguity of eigenvectors of a parahermitian $E V D)$ ): If $\boldsymbol{R}(z)$ has no identical eigenvalues, then there exist unique 1-d subspaces for analytic eigenvectors of a parahermitian matrix EVD, if and only if the eigenvalues are analytic across a potential algebraic multiplicity greater than one on the unit circle. Within this 1-d subspace, an eigenvector exists as a convergent Laurent or power series if its arbitrary phase response is selected such that the resulting eigenvectors are Hölder continuous in frequency $\Omega$.

Proof: It is known that the eigenvectors can be chosen to be analytic on the unit circle if and only if the eigenvalues are (e.g. Lemma 1). Each eigenvector $\boldsymbol{q}_{m}\left(\mathrm{e}^{\mathrm{j} \Omega}\right), m=1, \ldots, M$, can always be multiplied by an arbitrary phase response $\Phi_{m}\left(\mathrm{e}^{\mathrm{j} \Omega}\right)$, provided $\left|\Phi_{m}\left(\mathrm{e}^{\mathrm{j} \Omega}\right)\right|=1$ for all $\Omega$. If this phase response creates an eigenvector $\boldsymbol{q}_{m}\left(\mathrm{e}^{\mathrm{j} \Omega}\right)$ that is Hölder continuous for all $\Omega$, then $\boldsymbol{q}_{m}\left(\mathrm{e}^{\mathrm{j} \Omega}\right)$ can be represented by an absolutely convergent Fourier series as in (27). Analogous to the proof of Theorem 3, therefore an absolutely convergent power or Laurent series $\boldsymbol{u}_{m}(z)$ exists as the eigenvector, which matches $\boldsymbol{q}_{m}\left(\mathrm{e}^{\mathrm{j} \Omega}\right)$ on the unit circle, i.e. $\left.\boldsymbol{u}_{m}(z)\right|_{z=\mathrm{e}^{\mathrm{j} \Omega}}=\boldsymbol{q}_{m}\left(\mathrm{e}^{\mathrm{j} \Omega}\right)$. The selection of the phase response will have an impact on the causality of $\boldsymbol{u}_{m}(z)$, i.e. whether it will be a power or Laurent series.

If the phase response is selected more strictly such that $\boldsymbol{q}_{m}\left(\mathrm{e}^{\mathrm{j} \Omega}\right)$ is not just Hölder continuous but analytic, then an analytic $\boldsymbol{u}_{m}(z)$ can be obtained by analytic continuation via $z=\mathrm{e}^{\mathrm{j} \Omega}$ [29], [38], [39].

As a converse to Theorem 4, when eigenvalues are not selected analytic on the unit circle, e.g. by enforcing spectral majorisation in the case of an algebraic multiplicity of eigenvalues greater than one on the unit circle, or in the case of analytic eigenvalues but a discontinuous phase response $\Phi_{m}\left(\mathrm{e}^{\mathrm{j} \Omega}\right)$, discontinuous eigenvectors $\boldsymbol{q}_{m}\left(\mathrm{e}^{\mathrm{j} \Omega}\right)$ arise for which no exact representation by an absolutely convergent power or Laurent series exists.

\section{Approximation of Eigenvectors}

It is clear that if all eigenvectors $\boldsymbol{q}_{m}\left(\mathrm{e}^{\mathrm{j} \Omega}\right), m=1 \ldots M$ are Hölder continuous by virtue of analytic eigenvalues $\lambda_{m}\left(\mathrm{e}^{\mathrm{j} \Omega}\right)$ and appropriate phase responses, the convergent Laurent or power series $\boldsymbol{U}(z)$ can be approximated arbitrarily closely by Laurent polynomials $\hat{\boldsymbol{U}}(z)$-or polynomials in the case that the phase response admits a causal $\hat{U}(z)$ - analogously to (21). The speed of convergence depends on the smoothness of $\boldsymbol{Q}\left(\mathrm{e}^{\mathrm{j} \Omega}\right)$, with faster convergence for smoother functions. The fastest convergence can be expected if $\boldsymbol{Q}\left(\mathrm{e}^{\mathrm{j} \Omega}\right)$ is analytic, the conditions for which are given by Rellich [35] and highlighted in Lemma 1 (see Section V-B.)

For the following cases, Theorem 4 could not prove the existence of absolutely convergent power or Laurent series as eigenvectors $\boldsymbol{U}(z)$. Nevertheless, approximations may still be found:

- Discontinuous Phase Response. For analytic eigenvalues, as long as the phase response $\boldsymbol{q}_{m}\left(\mathrm{e}^{\mathrm{j} \Omega}\right)$ is piecewise continuous with a finite number of jump discontinuities, an 
approximation $\hat{\boldsymbol{U}}(z)$ can be reached via a Fourier series which on the unit circle converges to $\boldsymbol{Q}\left(\mathrm{e}^{\mathrm{j} \Omega}\right)$ except at these discontinuities. At discontinuities, the approximation $\left.\hat{\boldsymbol{U}}(z)\right|_{z=\mathrm{e}^{\mathrm{j} \Omega}}$ will converge to the average values stated in (28).

- Spectrally Majorised Eigenvalues. If eigenvalues have an algebraic multiplicity greater than one on the unit circle, discontinuities arise for the corresponding eigenvectors $\boldsymbol{q}_{m}\left(\mathrm{e}^{\mathrm{j} \Omega}\right)$. Provided that these, together with any discontinuities introduced by the phase responses, are finite in number, a polynomial or Laurent polynomial approximation $\hat{\boldsymbol{U}}(z)$ via a Fourier series obeying (28) can be found.

\section{NUMERICAL EXAMPLE}

We provide results for a numerical example with known ground truth for a PhEVD with both analytic and spectrally majorised eigenvalues, as well as for the results obtained by the SBR2 algorithm [6]. This informs observations on differences between the theoretical PhEVD established in terms of its existence and uniqueness in this paper, and what is obtainable via iterative polynomial EVD algorithms.

Consider $\boldsymbol{R}(z)=\boldsymbol{U}(z) \boldsymbol{\Gamma}(z) \boldsymbol{U}^{\mathrm{P}}(z)$ with paraunitary $\boldsymbol{U}(z)=$ $\left[\boldsymbol{u}_{1}(z), \boldsymbol{u}_{2}(z)\right]$ and $\boldsymbol{u}_{1,2}(z)=\left[1, \pm z^{-1}\right]^{\mathrm{T}} / \sqrt{2}$. With the diagonal and parahermitian $\Gamma(z)=\operatorname{diag}\left\{z+3+z^{-1} ;-\mathrm{j} z+3+\mathrm{j} z^{-1}\right\}$, the parahermitian matrix $\boldsymbol{R}(z): \mathbb{C} \rightarrow \mathbb{C}^{2 \times 2}$ is

$$
\boldsymbol{R}(z)=\left[\begin{array}{cc}
\frac{1-\mathrm{j}}{2} z+3+\frac{1+\mathrm{j}}{2} z^{-1} & \frac{1+\mathrm{j}}{2} z^{2}+\frac{1-\mathrm{j}}{2} \\
\frac{1+\mathrm{j}}{2}+\frac{1-\mathrm{j}}{2} z^{-2} & \frac{1-\mathrm{j}}{2} z+3+\frac{1+\mathrm{j}}{2} z^{-1}
\end{array}\right] .
$$

Analytic / Maximally Smooth Case. When extracting eigenvalues that are analytic on the unit circle, the solution is given by the diagonal elements of $\Gamma(z)=$ $\operatorname{diag}\left\{\left[z+3+z^{-1} ;-\mathrm{j} z+3+\mathrm{j} z^{-1}\right]\right\}$, which are taken from an example in [26]. The two eigenvalues overlap at $\Omega=\frac{1}{4} \pi$ and $\Omega=\frac{5}{4} \pi$, where they have an algebraic multiplicity of two, as shown in Fig. 5(a).

The two eigenvectors $\boldsymbol{u}_{1,2}(z)=\left[1, \pm z^{-1}\right]^{\mathrm{T}} / \sqrt{2}$ are of order one. To show that their evaluation on the unit circle evolves smoothly with frequency $\Omega$, we define $\varphi_{m}\left(\mathrm{e}^{\mathrm{j} \Omega}\right)$ as the Hermitian subspace angle [49], [50] relative to the arbitrary reference vector $\boldsymbol{u}_{1}\left(\mathrm{e}^{\mathrm{j} 0}\right)$,

$$
\cos \varphi_{m}\left(\mathrm{e}^{\mathrm{j} \Omega}\right)=\left|\boldsymbol{u}_{1}^{\mathrm{H}}\left(\mathrm{e}^{\mathrm{j} 0}\right) \boldsymbol{u}_{m}\left(\mathrm{e}^{\mathrm{j} \Omega}\right)\right|,
$$

with $m=1,2$ and $0 \leq \varphi_{m}\left(\mathrm{e}^{\mathrm{j} \Omega}\right) \leq \frac{\pi}{2}$. Similar to the subspace distance discussed in Section III-D, in the absence of an algebraic multiplicity of eigenvalues greater than one, these angles can be shown to evolve continuously under sufficiently small perturbations of $\mathbf{R}(z)$ [51]-[53].

Fig. 5(b) shows the subspace angles in (38), and indicates their smooth evolution with frequency. Note that because of the modulus operation involved in the Hermitian angles, the latter are reflected at $\varphi=0$ and $\varphi=\frac{\pi}{2}$, making $\varphi\left(\mathrm{e}^{\mathrm{j} \Omega}\right)$ nondifferentiable even though the eigenvectors themselves can be differentiated w.r.t. $\Omega$.

Ideal Spectral Majorisation. To achieve spectral majorisation, the eigenvalues of the analytic case have to be permuted on the frequency interval $\Omega=\left[\frac{1}{4} \pi, \frac{5}{4} \pi\right]$ as shown in Fig. 6(a).

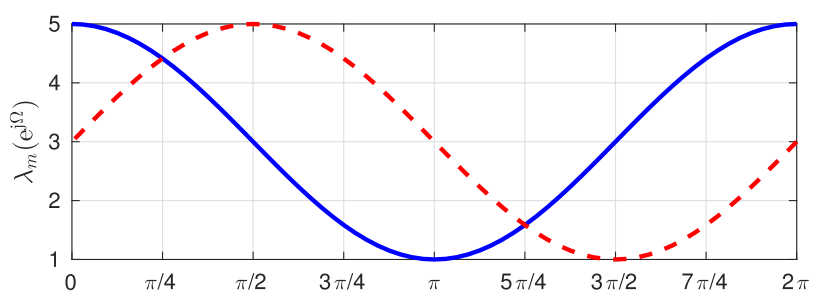

(a)

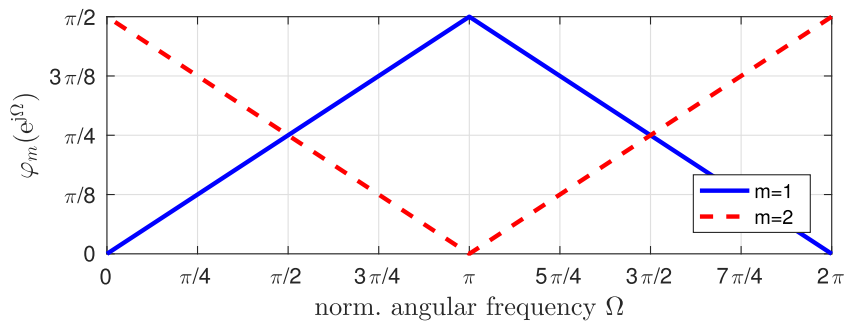

(b)

Fig. 5. (a) PSDs of eigenvalues that are analytic on the unit circle and (b) subspace angles of corresponding eigenvectors.

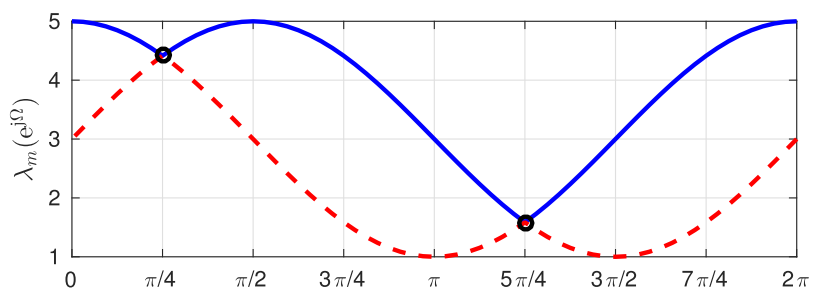

(a)

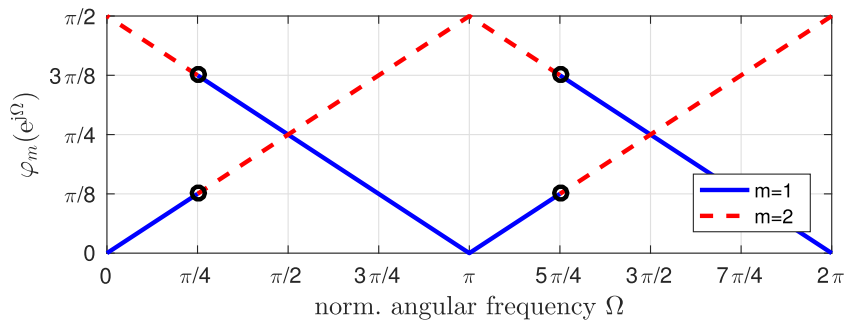

(b)

Fig. 6. (a) Ideally spectrally majorised eigenvalues and (b) subspace angle of corresponding discontinuous eigenvectors, defined on the unit circle; for the latter, no power series $\boldsymbol{u}_{m}(z)$ exists; black circles indicate points of nondifferentiability and discontinuities.

Note that the resulting PSDs are Hölder continuous but no longer differentiable at $\Omega=\frac{1}{4} \pi$ and $\Omega=\frac{5}{4} \pi$. As a consequence, the eigenvectors also must be permuted on the interval $\Omega=\left[\frac{1}{4} \pi, \frac{5}{4} \pi\right]$, which leads to discontinuous jumps of $\boldsymbol{q}_{m}\left(\mathrm{e}^{\mathrm{j} \Omega}\right)$ and subsequently the subspace angles at $\Omega=\frac{1}{4} \pi$ and $\Omega=\frac{5}{4} \pi$, as depicted in Fig. 6. For the Hölder continuous eigenvalues, unique convergent Laurent series $\gamma_{m}(z), m=1,2$ exist. However in contrast to the above maximally smooth case, for the eigenvectors, no absolutely convergent Laurent or power series $\mathbf{u}_{m}(z)$ matches $\mathbf{q}_{m}\left(\mathrm{e}^{\mathrm{j} \Omega}\right)$ on the unit circle.

Spectral Majorisation via SBR2. Applying the SBR2 algorithm [6] to $\boldsymbol{R}(z)$ in (37) should give polynomial approximations of the eigenvalues and eigenvectors characterised on the unit circle in Fig. 6 since SBR2 is proven to converge to a diagonal [6] and spectrally majorised parahermitian matrix [54]. 


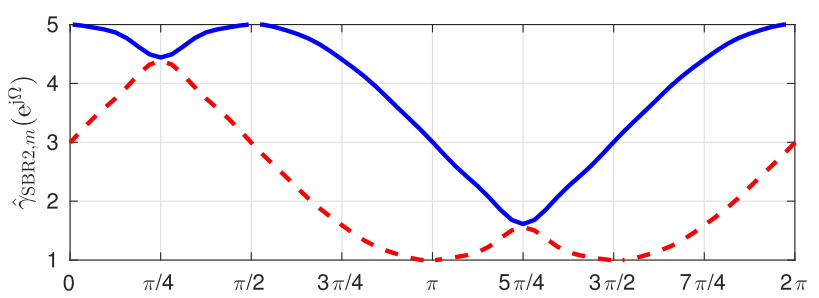

(a)

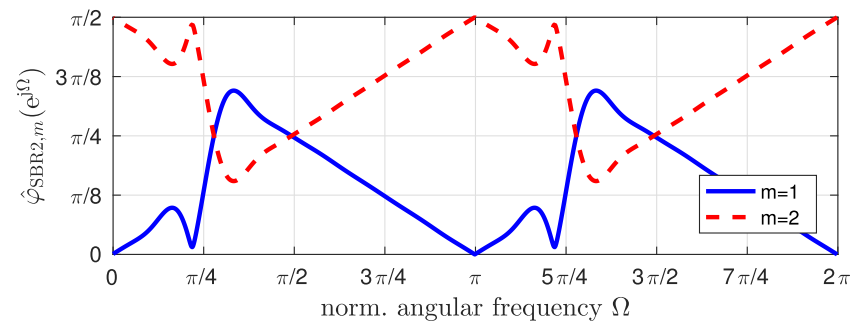

(b)

Fig. 7. (a) Approximate Laurent polynomial eigenvalues and (b) subspace angle of corresponding approximate Laurent polynomial eigenvectors obtained with the SBR2 algorithm [6] applied to $\boldsymbol{R}(z)$ in (37).

After 200 iterations and truncating small trailing values, SBR2 generates a polynomial approximation $\hat{\Gamma}(z)$ of the spectrally majorised eigenvalues in Fig. 7(a). Of order 24, these spectrally majorised eigenvalues approximate the ideal eigenvalues in Fig. 6(a) reasonably well, but are considerably longer than the PSDs of order 2 for the case of selecting eigenvalues that are analytic on the unit circle.

The paraunitary matrix obtained by SBR2 contains the polynomial approximations of the eigenvectors, with their subspace angles according to (38) shown in Fig. 7(b). Near the algebraic multiplicities at $\Omega=\frac{1}{4} \pi$ and $\Omega=\frac{5}{4} \pi$, the polynomial solution enforces smoothness, and approximates $\boldsymbol{Q}\left(\mathrm{e}^{\mathrm{j} \Omega}\right)$ as well as possible, approximately obeying (28) at the discontinuities and exhibiting Gibbs oscillations in their vicinity. Approximating these jumps requires a high polynomial order, which in this case is 84 , compared to the simple first order eigenvalues that are obtained in the maximally smooth case.

\section{DisCUSSION AND CONCLUSIONS}

For an analytic, positive semi-definite parahermitian matrix $\boldsymbol{R}(z)$ whose entries are Laurent polynomials or rational functions in $z$, this paper has established under which conditions there exists a parahermitian matrix EVD, $\boldsymbol{R}(z)=$ $\boldsymbol{U}(z) \boldsymbol{\Gamma}(z) \boldsymbol{U}^{\mathrm{P}}(z)$, with paraunitary $\boldsymbol{U}(z)$ and parahermitian diagonal $\boldsymbol{\Gamma}(z)$. Based on an EVD on the unit circle, $\boldsymbol{R}\left(\mathrm{e}^{\mathrm{j} \Omega}\right)=$ $\boldsymbol{Q}\left(\mathrm{e}^{\mathrm{j} \Omega}\right) \boldsymbol{\Lambda}\left(\mathrm{e}^{\mathrm{j} \Omega}\right) \boldsymbol{Q}^{\mathrm{H}}\left(\mathrm{e}^{\mathrm{j} \Omega}\right)$, which exists for all frequencies $\Omega$, this paper has investigated whether the frequency domain quantities $\boldsymbol{Q}\left(\mathrm{e}^{\mathrm{j} \Omega}\right)$ and $\boldsymbol{\Lambda}\left(\mathrm{e}^{\mathrm{j} \Omega}\right)$ admit representations by power or Laurent series that are absolutely convergent (at least on the unit circle), i.e. whether they can be expressed in the time domain, such that an exact match is achieved on the unit circle with $\left.\boldsymbol{\Gamma}(z)\right|_{z=\mathrm{e}^{\mathrm{j} \Omega}}=\boldsymbol{\Lambda}\left(\mathrm{e}^{\mathrm{j} \Omega}\right)$ and $\left.\boldsymbol{U}(z)\right|_{z=\mathrm{e}^{\mathrm{j} \Omega}}=\boldsymbol{Q}\left(\mathrm{e}^{\mathrm{j} \Omega}\right)$.

We have constrained $\Lambda\left(\mathrm{e}^{\mathrm{j} \Omega}\right)$ to be Hölder continuous in order for an absolutely convergent Fourier series to exist. We focus in particular on the cases that $\Lambda\left(\mathrm{e}^{\mathrm{j} \Omega}\right)$ is analytic and/or spectrally majorised. In either case, the eigenvalues in $\boldsymbol{\Gamma}(z)$ exist as unique, absolutely convergent Laurent series. If $\boldsymbol{\Lambda}\left(\mathrm{e}^{\mathrm{j} \Omega}\right)$ is analytic in $\Omega$, then additionally $\Gamma(z)$ is also analytic in $z$ within some region of convergence. If the eigenvalues in $\boldsymbol{\Lambda}\left(\mathrm{e}^{\mathrm{j} \Omega}\right)$ possess an algebraic multiplicity greater than one, i.e. the PSDs of eigenvalues intersect, then analyticity and spectral majorisation cannot be reconciled, and one or the other has to be chosen. Eigenvectors - the columns of $\boldsymbol{U}(z)$ - only exist as convergent Laurent or power series if the eigenvalues $\Lambda\left(\mathrm{e}^{\mathrm{j} \Omega}\right)$ are selected analytic and if an arbitrary phase response is chosen such that Hölder continuous eigenvectors result on the unit circle. If additionally phase responses create eigenvectors that are analytic in $\Omega, U(z)$ will be analytic in $z$ within some region of convergence. Eigenvectors do not exist as absolutely convergent power or Laurent series in the case of spectral majorisation in the presence of an algebraic multiplicity of eigenvalues in $\boldsymbol{\Lambda}\left(\mathrm{e}^{\mathrm{j} \Omega}\right)$ greater than one, or if their arbitrary phase response is discontinuous.

Eigenvalues can be arbitrarily closely approximated by Laurent polynomials $\hat{\Gamma}(z)$ of sufficiently high order. Faster convergence and therefore lower-order approximations are possible for analytic $\boldsymbol{\Lambda}\left(\mathrm{e}^{\mathrm{j} \Omega}\right)$ compared to a case where differentiability of $\Lambda\left(e^{j \Omega}\right)$ is violated to enforce spectral majorisation. For eigenvectors, where $\boldsymbol{U}(z)$ exists as an absolutely convergent power series, polynomial approximations $\hat{\boldsymbol{U}}(z)$ can be obtained by truncating $\boldsymbol{U}(z)$, and the approximation order depends on the smoothness of the arbitrary phase response: the smoother the phase that is selected, generally the lower the order of the polynomial approximation that can satisfy a given limit for the approximation error. Where an exact $\boldsymbol{U}(z)$ does not exist because of discontinuities of $\boldsymbol{Q}\left(\mathrm{e}^{\mathrm{j} \Omega}\right)$, a polynomial approximation is still possible, provided that the discontinuities-due to spectral majorisation in case of overlapping PSDs of eigenvalues and to discontinuities in the arbitrary phase response-are finite in number. However, such a polynomial approximation $\hat{\boldsymbol{U}}(z)$ will require much higher order than in the case of an analytic $\Lambda\left(\mathrm{e}^{\mathrm{j} \Omega}\right)$ and a smooth phase response of $\boldsymbol{Q}\left(\mathrm{e}^{\mathrm{j} \Omega}\right)$.

Almost all current polynomial EVD algorithms produce a factorisation $\boldsymbol{R}(z) \approx \hat{\boldsymbol{U}}(z) \hat{\boldsymbol{\Gamma}}(z) \hat{\boldsymbol{U}}^{\mathrm{P}}(z)$ that is spectrally majorised (or very nearly so) despite there being no explicit algorithmic step so to do. Recently it has been shown that by construction the SBR2 algorithm implicitly produces a spectrally majorised solution [54]. Spectral majorisation is desirable for a limited number of applications, where e.g. the coding gain maximisation [8] or the extraction of MIMO subchannels of ordered quality [15][17] matter. If the subspace decomposition of a parahermitian matrix is important, such as for angle of arrival estimation [18], [19] or beamforming applications [13], [14], [22], [23], then the eigenvectors are central but not guaranteed to exist as convergent power series. Polynomial EVD algorithms converge but generally obtain solutions with a very high order associated with high computational complexity; our results show that this is not an algorithmic problem but associated with the fundamental existence of a PhEVD. We hope that the findings of this paper can trigger the development of parahermitian matrix EVD algorithms akin to initial efforts in [9] that target the extraction of analytic eigenvalues, where eigenvectors can be guaranteed to 
exist as convergent power series and be approximated by much shorter polynomials than in the case of spectral majorisation.

\section{ACKNOWLEDGMENT}

The authors are very grateful to Marc Moonen of Katholieke Universiteit Leuven, Belgium, and Vanni Noferini of the University of Essex, U.K., for pointing them towards the analytic singular value decomposition and the work of Franz Rellich, respectively.

\section{REFERENCES}

[1] P. P. Vaidyanathan, Multirate Systems and Filter Banks. Englewood Cliffs, NJ, USA: Prentice-Hall, 1993.

[2] G. W. Stewart, "The decompositional approach to matrix computation," Comput. Sci. Eng., vol. 2, no. 1, pp. 50-59, Jan./Feb. 2000

[3] G. H. Golub and C. F. V. Loan, Matrix computations. 4th ed. Baltimore, MD, USA: The John Hopkins Univ. Press, 2013.

[4] J. G. McWhirter and P. D. Baxter, "A novel technique for broadband SVD," in Proc. 12th Annu. Workshop Adapt. Sensor Array Signal Process., Lexington, KY, USA, 2004.

[5] A. Tkacenko and P. P. Vaidyanathan, "Iterative greedy algorithm for solving the FIR paraunitary approximation problem," IEEE Trans. Signal Process., vol. 54, no. 1, pp. 146-160, Jan. 2006.

[6] J. G. McWhirter, P. D. Baxter, T. Cooper, S. Redif, and J. Foster, "An EVD algorithm for para-hermitian polynomial matrices," IEEE Trans. Signal Process., vol. 55, no. 5, pp. 2158-2169, May 2007.

[7] A. Tkacenko, "Approximate eigenvalue decomposition of para-hermitian systems through successive FIR paraunitary transformations," in Proc. IEEE Int. Conf. Acoust. Speech Signal Process., Dallas, TX, USA, Mar. 2010, pp. 4074-4077.

[8] S. Redif, J. G. McWhirter, and S. Weiss, "Design of FIR paraunitary filter banks for subband coding using a polynomial eigenvalue decomposition," IEEE Trans. Signal Process., vol. 59, no. 11, pp. 5253-5264, Nov. 2011.

[9] M. Tohidian, H. Amindavar, and A.M. Reza, "A DFT-based approximate eigenvalue and singular value decomposition of polynomial matrices," EURASIP J. Adv. Signal Process., vol. 2013, no. 1, pp. 1-16, 2013.

[10] J. Corr, K. Thompson, S. Weiss, J. G. McWhirter, S. Redif, and I. K. Proudler, "Multiple shift maximum element sequential matrix diagonalisation for parahermitian matrices," in Proc. IEEE Workshop Statist. Signal Process., Gold Coast, VIC, Australia, Jun. 2014, pp. 312-315.

[11] S. Redif, S. Weiss, and J. G. McWhirter, "Sequential matrix diagonalization algorithms for polynomial EVD of parahermitian matrices," IEEE Trans. Signal Process., vol. 63, no. 1, pp. 81-89, Jan. 2015.

[12] Z. Wang, J. G. McWhirter, J. Corr, and S. Weiss, "Multiple shift second order sequential best rotation algorithm for polynomial matrix EVD," in Proc. 23rd Eur. Signal Process. Conf., Nice, France, Sep. 2015, pp. 844848.

[13] S. Redif, J. G. McWhirter, P. D. Baxter, and T. Cooper, "Robust broadband adaptive beamforming via polynomial eigenvalues," in Proc. OCEANS, Boston, MA, USA, Sep. 2006, pp. 1-6.

[14] C. L. Koh, S. Redif, and S. Weiss, "Broadband GSC beamformer with spatial and temporal decorrelation," in Proc. 17th Eur. Signal Process. Conf., Glasgow, U.K., Aug. 2009, pp. 889-893.

[15] M. Davies, S. Lambotharan, and J. G. McWhirter, "Broadband MIMO beamforming using spatial-temporal filters and polynomial matrix decomposition," in Proc. 15th Int. Conf. Digit. Signal Process., Cardiff, U.K., Jul. 2007, pp. 579-582.

[16] C. H. Ta and S. Weiss, "A design of precoding and equalisation for broadband MIMO systems," in Proc. 15th Int.Conf. Digit. Signal Process., Cardiff, U.K., Jul. 2007, pp. 571-574.

[17] A. Sandmann, A. Ahrens, and S. Lochmann, "Resource allocation in SVDassisted optical MIMO systems using polynomial matrix factorization," in Proc. 16. ITG Symp. Photon. Netw., May 2015, pp. 1-7.

[18] M. Alrmah, S. Weiss, and S. Lambotharan, "An extension of the MUSIC algorithm to broadband scenarios using polynomial eigenvalue decomposition," in Proc. 19th Eur. Signal Process. Conf., Barcelona, Spain, Aug. 2011, pp. 629-633.

[19] S. Weiss, M. Alrmah, S. Lambotharan, J. G. McWhirter, and M. Kaveh, "Broadband angle of arrival estimation methods in a polynomial matrix decomposition framework," in Proc. IEEE 5th Int. Workshop Comput. Adv. Multi-Sensor Adapt. Process., Dec. 2013, pp. 109-112.
[20] P. G. Vouras and T. D. Tran, "Robust transmit nulling in wideband arrays," IEEE Trans. Signal Process., vol. 62, no. 14, pp. 3706-3719, Jul 2014.

[21] S. Weiss, S. Redif, T. Cooper, C. Liu, P. D. Baxter, and J. G McWhirter, "Paraunitary oversampled filter bank design for channel coding," EURASIP J. Appl. Signal Process., vol. 2006, pp. 1-10, 2006, Art. no. 31346 .

[22] A. Alzin, F. K. Coutts, J. Corr, S. Weiss, I. K. Proudler, and J. A. Chambers, "Polynomial matrix formulation-based Capon beamformer," in Proc. IMA Int. Conf. Signal Process. Math., Birmingham, U.K., Dec. 2016, pp. 1-4.

[23] S. Weiss, S. Bendoukha, A. Alzin, F. K. Coutts, I. K. Proudler, and J. A. Chambers, "MVDR broadband beamforming using polynomial matrix techniques," in Proc. 23rd Eur. Signal Process. Conf., Nice, France, Sep. 2015, pp. 839-843.

[24] S. Redif, S. Weiss, and J. G. McWhirter, "Relevance of polynomial matrix decompositions to broadband blind signal separation," Signal Process. vol. 134, pp. 76-86, May 2017.

[25] I. Gohberg, P. Lancaster, and L. Rodman, Matrix Polynomials. New York, NY, USA: Academic, 1982.

[26] S. Icart and P. Comon, "Some properties of Laurent polynomial matrices," in Proc. 9th IMA Conf. Math. Signal Process., Birmingham, U.K., Dec. 2012, pp. 1-4.

[27] D. Cescato and H. Bolcskei, "QR decomposition of laurent polynomial matrices sampled on the unit circle," IEEE Trans. Inf. Theory, vol. 56, no. 9 , pp. 4754-4761, Sep. 2010.

[28] D. Cescato and H. Bolcskei, "Algorithms for interpolation-based QR decomposition in MIMO-OFDM systems," IEEE Trans. Signal Process. vol. 59, no. 4, pp. 1719-1733, Apr. 2011.

[29] B. Girod, R. Rabenstein, and A. Stenger, Signals and Systems. Chichester, U.K.: Wiley, 2001

[30] T. Kato, "Die Grundlehren der mathematischen Wissenschaften in Einzeldarstellungen," in Perturbation Theory for Linear Operators, vol. 132 Berlin, Germany: Springer, 1966.

[31] G. W. Stewart and J.-g. Sun, Matrix Perturbation Theory. New York, NY, USA: Academic, 1990.

[32] J. L. Walsh, Interpolation and Approximation by Rational Functions in the Complex Domain. Providence, RI, USA: Amer. Math. Soc., 1965.

[33] A. Papoulis, Probability, Random Variables, and Stochastic Processes. 3rd ed. New York, NY, USA: McGraw-Hill, 1991.

[34] A. V. Oppenheim, R. W. Schafer, and J. R. Buck, Discrete-Time Signal Processing, 2nd ed. London, U.K.: Pearson, 1999.

[35] F. Rellich, "Störungstheorie der Spektralzerlegung. I. Mitteilung. Analytische Störung der isolierten Punkteigenwerte eines beschränkten Operators," Mathematische Annalen, vol. 113, pp. DC-DCXIX, 1937.

[36] A. Tkacenko and P. P. Vaidyanathan, "On the spectral factor ambiguity of FIR energy compaction filter banks," IEEE Trans. Signal Process., vol. 54, no. 1, pp. 380-385, Jan. 2006.

[37] A. Kirac and P. P. Vaidyanathan, "Theory and design of optimum FIR compaction filters," IEEE Trans. Signal Process., vol. 46, no. 4, pp. $903-$ 919, Apr. 1998.

[38] L. V. Ahlfors, Complex analysis: An Introduction to the Theory of Analytic Functions of one Complex Variable. New York, NY, USA: McGraw-Hill, 1953.

[39] H. Behnke and F. Sommer, Theorie der Analytischen Funktionen Einer Komplexen Veränderlichen. Berlin, Germany: Springer, 1955.

[40] E. M. Stein and R. Shakarchi, Fourier Analysis: An Introduction. Princeton, NJ, USA: Princeton Univ. Press, 2003.

[41] K. Weierstrass, "Über die analytische Darstellbarkeit sogenannter willkürlicher Functionen einer reellen Veränderlichen. Erste Mittheilung," Sitzungsberichte der Königlich Preußischen Akademie der Wissenschaften zu Berlin, Jul. 1885, pp. 633-639.

[42] K. Weierstrass, "Über die analytische Darstellbarkeit sogenannter willkürlicher Functionen einer reellen Veränderlichen. Zweite Mittheilung," Sitzungsberichte der Königlich Preußischen Akademie der Wissenschaften zu Berlin, Jul. 1885, pp. 789-805.

[43] A. J. Hoffman and H. W. Wielandt, "The variation of the spectrum of a normal matrix," Duke Math. J., vol. 20, pp. 37-39, 1953.

[44] B. L. R. De Moor and S. P. Boyd, "Analytic properties of singular values and vectors," Katholic Univ. Leuven, Belgium Tech. Rep. 1989-28, 1989.

[45] A. Bunse-Gerstner, R. Byers, V. Mehrmann, and N. K. Nicols, "Numerical computation of an analytic singular value decomposition of a matrix valued function," Numer. Math, vol. 60, pp. 1-40, 1991.

[46] C. D. Meyer and G. W. Stewart, "Derivatives and perturbations of eigenvectors," SIAM J. Numer. Anal., vol. 25, no. 3, pp. 679-691, Jun. 1988.

[47] M. H. Stone, "The generalized Weierstrass approximation theorem," Math. Mag., vol. 21, no. 4, 5, pp. 167-184, pp. 237-254, Mar.-Jun. 1948. 
[48] J. Corr, K. Thompson, S. Weiss, I. K. Proudler, and J. G. McWhirter, "Impact of source model matrix conditioning on PEVD algorithms," in Proc. IET/EURASIP Intell. Signal Process., London, U.K., Dec. 2015, pp. 1-6.

[49] E. Study, "Kürzeste Wege im komplexen Gebiet," Mathematische Annalen, vol. 90, pp. 321-377, 1905.

[50] K. Scharnhorst, "Angles in complex vector spaces," Acta Applicandae Mathematica, vol. 69, no. 1, pp. 95-103, 2001.

[51] C. Davis and W. M. Kahan, "The rotation of eigenvectors by a perturbation. III," SIAM J. Numer. Anal., vol. 7, no. 1, pp. 1-46, 1970.

[52] G. W. Stewart, "Error bounds for approximate invariant subspaces of closed linear operators," SIAM J. Numer. Anal., vol. 8, no. 4, pp. 796-808, 1971.

[53] J.-g. Sun, "Perturbation of angles between linear subspaces," J. Comput. Math., vol. 5, no. 1, pp. 58-61, 1987.

[54] J. G. McWhirter and Z. Wang, "A novel insight to the SBR2 algorithm for diagonalising para-hermitian matrices," in Proc. 11th IMA Conf. Math. Signal Process., Birmingham, U.K., Dec. 2016, pp. 1-4.

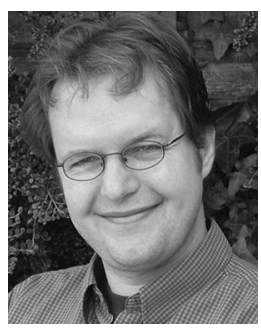

Stephan Weiss (S'97-A'98-M'99-SM'05) received the Dipl.-Ing. degree from the University of ErlangenNürnberg, Erlangen, Germany, in 1995, and the $\mathrm{Ph} . \mathrm{D}$. degree from the University of Strathclyde, Glasgow, U.K, in 1998, both in electronic and electrical engineering.

$\mathrm{He}$ is a Professor and the Head of the Centre for Signal and Image Processing with the University of Strathclyde, following previous academic appointments with the University of Strathclyde and the University of Southampton, Southampton, U.K. He has authored or co-authored more than 280 technical papers. His research interests include adaptive, multirate, and array signal processing with applications in communications, audio, and biomedical signal processing

Dr Weiss was a co-recipient of the 2001 Research Award of the German society on hearing aids in biomedical signal processing and the VTC-Spring Best Paper Award in the MIMO systems track in 2011. He is a member of EURASIP He was the Technical Co-chair for EUSIPCO 2009 and the General Chair of IEEE ISPLC 2014, both organised in Glasgow, U.K. He is an Associate Editor for Elsevier Digital Signal Processing, and previously served on the editorial board of IEEE TRANSACTIONS ON MOBILE COMPUTING.

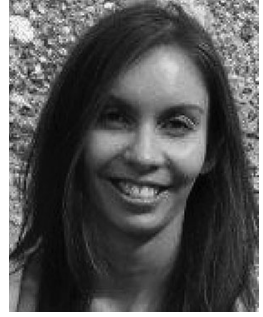

Jennifer Pestana received the Bachelor of Mathematics degree in 2006 and the Bachelor of Applied Science (Hons.) degree in 2007 from the Queensland University of Technology, Brisbane, QLD, Australia. She received the D.Phil. in numerical analysis from the University of Oxford, Oxford, U.K., in 2012. During 2012-2013, she was a Postdoctoral with the University of Oxford and the University of Manchester during 2014-2015. Since 2015, he has been a Lecturer with the University of Strathclyde, Glasgow, U.K. Her research interests include problems in numerical linear algebra and scientific computing.



Ian K. Proudler received the Graduate degree in physics from the Oxford University, Oxford, U.K., in 1978 and the Ph.D. degree in digital signal processing from the University of Cambridge, Cambridge, U.K., in 1984.

He spent two years doing R\&D work in the electronics industry. He is currently a Visiting Professor of signal processing with the University of Strahclyde, Glasgow, U.K. From 1986 to 2011, he was with the defence sector looking into various adaptive digital signal processing issues, such as numerical stability and efficient computation, antenna algorithm for HF communications, signal separation for ESM purposes, magnetic detection for maritime surveillance, and GPS antijam systems. He has authored or co-authored more than 100 research papers, contributed to 4 textbooks and holds a patent on an adaptive filtering architecture.

Dr. Proudler was the recipient of the John Benjamin Memorial Prize, in 1992 and 2001, and the IEE J.J. Thomson Medal, in 2002, for his work on signal processing algorithms. He was an Honorary Editor for IEE Proceedings: Radar, Sonar and Navigation for ten years. He has been on the organizing committee of several international conferences. 